\title{
Modeling solute transport and mixing in heterogeneous porous media under turbulent flow conditions
}

Elisa Baioni, ${ }^{1,2}$ Mohaddeseh Mousavi Nezhad, ${ }^{1}$ Giovanni Michele Porta, ${ }^{2}$ and Alberto Guadagnini ${ }^{2}$
${ }^{1)}$ School of Engineering, University of Warwick, Coventry CV47AL, UK

${ }^{2}$ Dipartimento di Ingegneria Civile e Ambientale, Politecnico di Milano, Milano 20133,

Italy

(*Electronic mail: alberto.guadagnini@ polimi.it)

(*Electronic mail: giovanni.porta@polimi.it)

(*Electronic mail: M.Mousavi-Nezhad@warwick.ac.uk)

(*Electronic mail: e.baioni@warwick.ac.uk or elisa.baioni@polimi.it)

(Dated: 15 September 2021)

We develop and test a modeling approach to quantify turbulence-driven solute transport and mixing in porous media. Our approach addresses two key elements: $(a)$ the spatial variability of the effective diffusion coefficient which is typically documented in the presence of a sediment-fluid interface and $(b)$ the need to provide a model that can yield the complete distribution of the concentration probability density function, not being limited only to the mean concentration value and thus fully addressing solute mixing. Our work is motivated by the importance of solute transport processes in the hyporheic zone, which can have strong implications to natural attenuation of pollutants. Our approach combines Lagrangian schemes to address transport and mixing in the presence of spatial variability of effective diffusion. An exemplary scenario we consider targets a set-up constituted by a homogeneous (fully saturated) porous medium underlying a clear water column where turbulent flow is generated. Solute concentration histories obtained through a model based solely on diffusive transport are benchmarked against an analytical solution. These are then compared against the results obtained by modeling the combined effects of diffusion and mixing. A rigorous sensitivity analysis is performed to evaluate the influence of model parameters on solute concentrations and mixing, the latter being quantified in terms of the scalar dissipation rate.

\section{INTRODUCTION}

Reliable modeling of mass and momentum transport at the interface between free flow and a porous medium is key to enhance our understanding of the functioning of aquatic systems and their interaction with the groundwater compartment. A notable example in this context is the hyporheic zone, which corresponds to the portion of the stream bed characterized by the mixing between surface water and groundwater. This region significantly contributes to mitigation of water pollution through a combined effect of bio-degradation, mixing, and sorption/desorption processes ${ }^{1,2}$. Mass and flow exchange between groundwater and the free surface stream drives pollutants into the bed sediment, where these are temporarily retained and can be subject to transformation by chemical reactions. Such interaction is typically termed as hyporheic exchange and takes place mainly across the region close to the water-sediment interface (i.e., the benthic biolayer). Hyporheic solute exchange is driven by physical and chemical gradients ${ }^{3}$, molecular diffusion, turbulent shear-driven flow, and advective phenomena arising in the presence of river bedforms ${ }^{4}$

Quantification of the relative contributions of the abovementioned processes on solute exchange at the sedimentwater interface is challenging also considering the natural variability of physical attributes of sediments. The distinction between transport and mixing processes driving solute migration across a porous medium is an important element to consider in this context. While modeling of transport mainly focuses on the assessment of volume averaged concentration values, mixing is typically defined as the ensemble of processes favoring a scenario where two substances initially residing into diverse volumes end up occupying the same (common) volume ${ }^{5,6}$. Mixing and transport features in natural systems are strongly interrelated and are affected by heterogeneities of system attributes/properties which are distributed across a variety of scales ${ }^{7}$. From a physical standpoint, advection and molecular diffusion are key drivers of dissolved solute particles. While (spatially) heterogeneous advection leads to solute spreading, diffusion contributes to dilution, ultimately leading to spatial homogenization of solute concentrations $^{7,8}$.

In porous media, solute mixing processes acting at the pore scale are often depicted by coupling the effects of advection and diffusion within a lumped dispersion coefficient whose formulation can be obtained through upscaling of pore-scale information to the Darcy (or continuum) scale. This approach is documented to suffer from neglecting the nonlinear nature of mixing processes and leads to an inaccurate evaluation of local concentrations 6,9 . With reference to the characterization of transport processes at sediment-water interfaces, experimental tests have been performed to improve our understanding of mass transport and of the way turbulence effects within a porous medium affect solute arrival times at locations of interest ${ }^{10-15}$. In this context, a commonly used approach relies on characterizing transport by means of an effective diffusion coefficient. Recent results show that the effective diffusion coefficient exhibits an exponential reduction with depth below the exchange interface ${ }^{10}$, this pattern having 
strong effects on solute transport and mixing even in seemingly homogeneous porous domains. To overcome the limitations of available approaches, our study targets the quantification of the effect of porous medium heterogeneity on global mixing metrics. While the impact on mixing of spatially heterogeneous flow properties has been widely investigated in the literature ${ }^{9}$, diffusion coefficients are often considered as constant in space ${ }^{16,17}$. For example, the majority of the models employed in practical settings to quantify solute exchange across the hyporheic zone neglects spatial heterogeneity of diffusion coefficients and typically relies on a simplified description of transport and mixing in the pore space ${ }^{18-26}$. Results from computational studies ${ }^{27}$ suggest that neglecting the spatial variation of diffusion parameters leads to an underestimation of solute concentrations across a porous system. Here, we focus on two main processes: $(i)$ diffusive solute transport caused by macroscopic concentration gradients and (ii) mixing induced by molecular diffusion enhanced by turbulent oscillations of the velocity field at the pore scale.

We model these processes through a random walk technique that relies on two main elements: $(i)$ the Skew Brownian Motion (SBM) model ${ }^{28}$ and (ii) the Parameterized scalar profile (PSP) mixing model ${ }^{29}$. The SBM model ${ }^{28}$ is used to assess the behavior of solute particles across media characterized by a spatially variable (and piece-wise constant) diffusion coefficient. The choice of this approach is motivated by the possible occurrence of sharp (spatial) variations in the system diffusivity that may arise due to physical heterogeneity of the porous system or to the decrease in the strength of turbulence effects which is documented to take place in the porous domain close to a sediment-water interface. To address mixing, we employ a modified version of the PSP mixing model $^{29}$ that enables the evaluation of the temporal evolution of chemical concentration and mixing of solute mass within the fluid domain. The implemented approach allows approximating the dynamics of the full probability density function (PDF) of solute concentration in the domain, and thereby compute global indicator of mixing, such as the scalar dissipation rate ${ }^{30}$. The approach is here applied to two settings: $(a)$ a bimaterial medium, consisting of two sediment layers, subject to a diffusive gradient; and $(b)$ a system inspired by the experimental setup considered in Chandler et al. ${ }^{10}$. In the context of the latter scenario, we explore the sensitivity of the model outputs to uncertainty associated with input parameters employed to mode the space-time evolution of solute concentrations and metrics employed to characterize mixing. Our analysis is keyed to the identification of the main parameters driving mixing-driven reactive processes.

The work is structured as follows. Section II is devoted to illustrate the methodology at the core of the modeling approach: the SBM model ${ }^{28}$ is introduced in Section II A while the PSP mixing model $^{29}$ is presented in Section II B. The key results of the study are shown in Section III for case $(a)$ (Section III A) and case $(b)$ (Section III B). Conclusions are then presented in Section IV.

\section{METHODOLOGY}

Our study targets diffusive transport and mixing of chemicals in the presence of sharp spatial variations of the system effective diffusivity of the kind that can take place close to a sediment-water interface. We rely on coupling two existing models based on a Lagrangian perspective, i.e., $(i)$ the Skew Brownian Motion (SBM) model ${ }^{28}$, which is typically employed to assess diffusive transport in the presence of a spatially heterogeneous diffusion coefficient, and (ii) the parameterized scalar profile (PSP) mixing model ${ }^{29}$. Some details on these approaches are provided in Section II A and Section II B, Section II C describing our strategy to couple them.

\section{A. Skew Brownian Motion (SBM) model}

Assessment of scenarios driven by diffusive gradient conditions is challenging. The Skew Browian Motion (SBM) model was introduced in the 70 by Itô ${ }^{31}$ and Walsh ${ }^{32}$ as a generalization of the Brownian motion ${ }^{33}$. The approach introduced in Lejay and Pichot ${ }^{28,34}$ is then used to treat diffusive problems in media with spatially discontinuous values of the diffusion coefficient. We start by considering a simple case where a one-dimensional domain is composed by two regions, each characterized by a constant diffusion coefficient, i.e., we set $D=D^{+}$for $X>X_{I}$ and $D=D^{-}$for $X<X_{I}$, where $X_{I}$ defines the location of the interface between the two regions and $X \in(-\infty,+\infty)$. The displacement of a given solute particle is depicted through the SBM only in the proximity of the discontinuity/interface $\left(X_{I}\right)$, its dynamics being otherwise modeled as a classical Brownian motion. The extent $d_{\text {layer }}$ of the region around the discontinuity (termed as interface layer) where the particle is affected by the variation of the diffusion coefficient is estimated by Eq. (1) for each portion of the domain with constant diffusion coefficient (i.e., $D^{ \pm}$)

$$
d_{\text {layer }}=c_{\alpha} \sqrt{2 D^{ \pm} \delta t_{t}}
$$

where $c_{\alpha}$ is an arbitrary constant and $\delta t_{t}$ is a fixed time step. As an example, considering a Gaussian distribution and assuming $c_{\alpha}=4$ (corresponding to $\alpha=1-6 \times 10^{-5}=99.994 \%$ ) the probability that a particle located outside the interface layer (i.e., at $x<X_{I}-c_{\alpha} \sqrt{2 D^{-} \delta t_{t}}$ or $x>X_{I}+c_{\alpha} \sqrt{2 D^{+} \delta t_{t}}$ (see Figure 1)) may reach the discontinuity at $X_{I}$ is equal to $0.006 \%$. Therefore, the domain is subdivided into the three diverse zones $^{34}$ (see Figure 1) detailed in the following.

1. Constant diffusion zone $\left(X_{b}+c_{\alpha} \sqrt{2 D^{-} \delta t_{t}}<x<X_{I}-\right.$ $\left.c_{\alpha} \sqrt{2 D^{-} \delta t_{t}}\right)$. A particle in this region is far enough from $X_{I}$ and is not influenced by the discontinuity. The particle motion is treated as a conventional diffusion scenario through

$$
x\left(t+\delta t_{t}\right)=x(t)+\xi \sqrt{2 D[x(t)] \delta t_{t}}
$$

where $x(t)$ is the particle position at time $t$ and $\xi$ is a random number sampled from a standard normal distribution; 
2. Interface layer $\left(X_{I}-c_{\alpha} \sqrt{2 D^{-} \delta t_{t}}<x<\right.$ $X_{I}+c_{\alpha} \sqrt{2 D^{+} \delta t_{t}}$ ). It is the area across the interface $X_{I}$ where the random walker is affected by the diffusive discontinuity and is displaced according to the SBM model;

3. Boundary layer $\left(X_{b}<x<X_{b}+c_{\alpha} \sqrt{2 D^{-} \delta t_{t}}\right)$. The behavior of a particle close to the domain boundary (located at $X_{b}$ ) is related to the imposed boundary conditions, reflecting boundaries being assumed in our study.

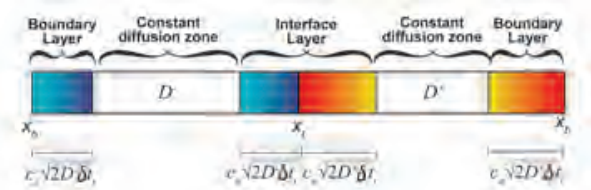

FIG. 1. Partition of the domain in three zones: Constant diffusion zone, interface layer, boundary layer

We focus here on the particles close to the interface $X_{I}$ and consider particle motion driven by the $S B M$ according to the following two steps which are implemented in sequence ${ }^{34}$ i.e., we $(i)$ first assess if the interface location is reached and then (ii) compute the particle position at time $t+\delta t_{t}$ based on the results obtained at step $(i)$. We consider the Exact Hitting Time algorithm ${ }^{34}$ to assess whether the particle crosses the interface and evaluate the time $\tau$ (termed first hitting time) required for the particle to move from its current position $X(t)$ to $X_{I}$. The algorithm is grounded on the concept of the Brownian bridge and is here employed as it provides a more accurate estimate of the first hitting time when compared to a linear approximation $^{34}$.

When the interface is not crossed within the considered time interval $\delta t_{t}$, the particle position at time $t+\delta t_{t}$ is directly calculated $^{34}$. Otherwise, if the interface is crossed, the particle is set to start moving from $X_{I}$ after a pre-defined time $\tau$ and step (ii) is then initiated. In other words, at time $t+\tau$ the particle/walker is still at the interface location. Once the particle has reached $X_{I}$, two possible scenarios may take place, i.e., either the interface is crossed or the particle is reflected The probability of a walker to cross or to be reflected once it reaches the interface is given by ${ }^{34}$

$$
\theta=\frac{\sqrt{D^{+}}-\sqrt{D^{-}}}{\sqrt{D^{+}}+\sqrt{D^{-}}}
$$

which essentially expresses the fraction of the mass flux that must be reflected at the discontinuity.

A random value is then drawn from a uniform distribution defined on the unit support and is compared against $\frac{1+\theta}{2}$ to assess if the particle is allowed to cross the boundary. We recall that the algorithm can be readily extended to settings associated with the presence of multiple discontinuities, as the motion of each particle only depends on its immediate surroundings ${ }^{28}$.

\section{B. PSP mixing model}

The parameterized scalar profile (PSP) ${ }^{29,35}$ is grounded on the idealization of a scalar field evolving across a turbulent flow domain as an ensemble of one-dimensional parameterized scalar profiles (PSP). For instance, the scalar can represent a temperature or, as in our study, the concentration of a chemical compound. Modeling mixing through scalar profiles is a classical approach underpinning the concept of continuum-scale mixing in a variety of models ${ }^{36-38}$. In the presence of a stationary homogeneous turbulence, the scalar value $\phi^{i}$ associated with each particle $p_{i}$ evolves to the local scalar mean $\langle\phi\rangle$ with a constant rate over time ${ }^{29}$. Mixing induces temporal evolution of the scalar attributed to each walker. The temporal variation of $\phi^{i}$ associated with particle $p_{i}$ is quantified by ${ }^{35}$

$$
\frac{d \phi^{i}}{d t}=-\frac{1}{\bar{\tau}_{\phi}}\left(\phi^{i}-\langle\phi\rangle\right)
$$

The mixing time scale $\bar{\tau}_{\phi}$ can be quantified as ${ }^{39}$

$$
\bar{\tau}_{\phi}=\frac{2}{C_{\phi}^{\prime}\langle w\rangle}
$$

where, $\langle w\rangle$ is a mean turbulence frequency and $C_{\phi}{ }^{\prime}$ is a constant model parameter that correlates such frequency to scalar mixing 40

The shape of the PDF of the considered scalar is preserved in spatially homogeneous turbulence settings because all particles of the ensemble tend to relax to the mean value $\langle\phi\rangle$ with the same rate (Eq. (4)). In the PSP mixing model, the onedimensional profile associated with each particle is approximated with a sinusoidal shape and is parametrized in terms of four quantities, i.e., $\phi_{+}, \phi_{-}, \lambda$, and $\bar{\tau}_{\phi}$, representing the largest and lowest bound of the range of values which can possibly be undertaken by the scalar (here termed as scalar boundary values), the profile length scale, and the mixing time scale, respectively. The sinusoidal profile is expressed as

$$
\phi^{i}(x, t)=e^{\left(-\frac{t}{\tau_{\phi}}\right)} \frac{\left(\phi_{+}{ }^{i}+\phi_{-}{ }^{i}\right)}{2} \sin \left(\frac{\pi}{\lambda^{i}} x\right)+\phi_{c}{ }^{i}
$$

where, $\phi_{c}{ }^{i}=\frac{\phi_{+}{ }^{i}+\phi_{-}{ }^{i}}{2}$ is the profile center.

Here, we consider the scalar field to correspond to solute concentration across the domain. Its dynamics are then quantified as

$$
\frac{d \phi^{i}}{d t}=D \frac{\partial^{2} \phi^{i}}{\partial x^{2}}
$$

where $x$ is the (one-dimensional) spatial profile coordinate, $D$ is the diffusion coefficient, and $\phi^{i}$ scalar attributed to the particle $p_{i}$. 
Combining Eqs. (4) and (7) yields

$$
\lambda=\pi \sqrt{D \tau_{\phi}}=\pi \sqrt{\frac{2 D}{C_{\phi}^{\prime} w}}
$$

We then rely on Eq. (8) and modify the original PSP model to assess the temporal evolution of the particle concentration

$$
\phi^{i}\left(t+\delta t_{m}\right)=\left(e^{-\frac{\pi^{2} D}{\lambda_{i}^{2}} \delta t_{m}}-1\right)\left(\phi^{i}(t)-\phi_{c}^{i}(t)\right)+\phi^{(i)}(t)
$$

where $\delta t_{m}$ is a time step employed to discretize the mixing process.

We note that our parameterization of the PSP model slightly differs from Meyer's original approach ${ }^{29,35}$ in that the profile length scale $\lambda$ is here considered as an input model parameter, as opposed to $C_{\phi}^{\prime} 29,35$. Our choice is consistent with the observation that we are considering mixing caused by the combined effect of diffusion and hydrodynamic dispersion in a porous medium, while Meyer ${ }^{29,35}$ tackles a homogeneous turbulen flow scenario. Therefore, a diverse value of $\lambda$ can be linked to each of the considered particles, due to the occurrence of possible spatial inhomogeneities in the turbulence characteristics across the pore space. Closure of model (9) requires specifying the value $\phi_{c}$, which is typically taken as the average between $\phi_{+}$and $\phi_{-}{ }^{29,35}$. The PDF of concentration at a given time within each cell of the domain is defined by the distribution of the values of $\phi^{i}$ (including $\phi_{+}$and $\phi_{-}$) associated with each particle $p_{i}$ within the cell ${ }^{29,35}$. For a given particle $p_{i}$ a pair of particles (termed as boundary particles and denoted as $i_{-}$and $i_{+}$) is randomly selected in the same cell so as to satisfy

$$
\left(\phi^{i}-\phi_{-}^{i}\right)\left(\phi^{i}-\phi_{+}^{i}\right) \leq 0
$$

which states that the scalar value $\phi^{i}$ of particle $p_{i}$ on a given profile should always be comprised between the boundary particle values.

Either of the boundary particle values can be updated when:

1. one of the two boundary particles leaves the current cell;

2. particle $p_{i}$ leaves the current cell; or

3. the variable time $t_{ \pm}$associated with either boundary particle is null or negative; note that $t_{ \pm}$decreases over time according to

$$
t_{ \pm}\left(t+\delta t_{m}\right)=t_{ \pm}(t)-\delta t_{m}
$$

where $t_{ \pm}\left(t+\delta t_{m}\right)$ and $t_{ \pm}(t)=\frac{\lambda_{ \pm}^{2}}{\frac{C_{t}}{c_{\phi}^{\prime}} 2 D_{k}}$ are the values associated with $t_{ \pm}$for $\phi_{+}, \phi_{-}$at time $t+\delta t_{m}$ and $t$, respectively; $D_{k}$ is the diffusion coefficient of layer $k$; and $\lambda_{ \pm}$is the length scale of profile in Eq. (6) for particles associated with $\phi_{+}, \phi_{-}$.
In case the replacement of particle $p_{i}$ is required, the corresponding scalar boundary values $\left(\phi_{+}^{i}\right.$ or $\left.\phi_{-}^{i}\right)$ is replaced by the particle residing within the same grid cell as particle $p_{i}$ and whose associated scalar value is closest to the one that is replaced.

\section{Coupling the SBM and PSP mixing models}

We consider transport and mixing in a layered domain, structured across $N_{L}$ layers, each characterized by a given diffusion coefficient. The approach proposed in the present work rests on coupling the SBM and the PSP mixing models. We recall that particles in the SBM model represent a mass of solute while representing an ensemble of concentration values in the PSP model. Coupling of the two approaches requires a mass-concentration conversion to ensure mass conservation. Particles are initially displaced according to the SBM model, each particle $p_{i}$ being associated with a given solute mass. Each particle is then related to a concentration value prior to applying the mixing model according to

$$
\phi_{i}=\frac{m_{i}}{V_{i}(k)}
$$

where $V_{i}=\frac{V_{L}(k)}{n_{L}(k)}$ is evaluated as the ratio between the fluid volume within layer $k$ (i.e., $V_{L}(k)$ ) where particle $p_{i}$ resides at time $t$ and the number of particles within the layer, $n_{L}(k)$ (with $1 \leq k \leq N_{L}$ ).

The mass of each particle is preserved during diffusive transport, i.e., $m_{i}\left(t+\delta t_{m}\right)=m_{i}(t)$. Otherwise, the layer $k$ where a given particle can be found may change in time. Eq. (12) can then be recast as

$$
\phi_{i}\left(t+\delta t_{m}\right)=\phi_{i}(t) \frac{V_{L}[k(t)]}{V_{L}\left[k\left(t+\delta t_{m}\right)\right]} \frac{n_{L}\left[k\left(t+\delta t_{m}\right)\right]}{n_{L}[k(t)]}
$$

The scalar values attributed to each of the walkers are rescaled in the event these are higher than the largest concentration observed in the system at the initial time. Rescaling is performed according to the principle that solute concentration cannot be higher than the initial conditions in the settings we analyze (i.e., in the absence of solute influx from the boundaries). Mixing is then simulated in accordance with the PSP mixing model, yielding a temporal variability of the concentration associated with each walker. Note that mixing is here considered to take place exclusively between walkers located within the same layer. The scalar values related to the particles are then converted into mass by

$$
m_{i}=\phi_{i} \frac{V_{L}(k)}{n_{L}(k)}
$$

and are employed in the subsequent step involving application of the SBM model. 


\section{Quantities of interest}

Our simulations are geared to analyzing three key output quantities: $(a)$ the average solute concentration $(\bar{C}),(b)$ the probability density function (PDF) of concentration values, and $(c)$ the spatial integral of the concentration squared, which is employed as the primary indicator of mixing across the entire domain. We consider transport in closed physical domains with time invariant properties. The average concentration is then constant in time and can be computed by a volumeweighted average of concentrations in each layer $C_{k}$ as

$$
\bar{C}=\frac{\sum_{k=1}^{N_{L}} C_{k} V_{L}(k)}{\sum_{k=1}^{N_{L}} V_{L}(k)}
$$

The mean solute concentration of each layer $k$ is evaluated as the average value of the scalars $\phi_{i}$ associated with the particles residing within the layer at a given time. Consistent with the procedure used for the evaluation of $\bar{C}$, the concentration PDF (within the domain at a given time) can be approximated as

$$
\bar{f}\left(\phi_{i}\right)=\frac{\sum_{k=1}^{N_{L}} f\left(\phi_{i}, k\right) V_{L}(k)}{\sum_{k=1}^{N_{L}} V_{L}(k)}
$$

where $f\left(\phi_{i}, k\right)$ is the PDF associated with the values of $\phi_{i}$ for particles that are in a given layer $k$. The integral of the squared concentration $\bar{C}^{2}$ is approximated as

$$
\overline{C^{2}}=\sum_{k=1}^{N_{L}} C_{k}^{2} V_{L}(k)
$$

where $C_{k}^{2}$ is defined as

$$
C_{k}^{2}=\frac{\sum_{i=1}^{n_{L}} \phi_{i}^{2}}{n_{L}}
$$

In addition to the three metrics described above, mixing dynamics in the whole system are also quantified in terms of the scalar dissipation rate, evaluated as ${ }^{9}$

$$
\chi(t)=-\frac{1}{2} \frac{d \bar{C}^{2}}{\delta t}
$$

\section{RESULTS AND DISCUSSION}

The following section is devoted to the illustration of the major results of the study. Two systems with a different degree of complexity are assessed:

- Case 1: a system structured across two layers, each characterized by a given diffusion coefficient (hereafter termed as bimaterial medium);
- Case 2: a system mimicking an erosimeter, consisting by a porous medium underlying a water column ${ }^{41,42}$. The porous medium is characterized by an exponential reduction of the diffusion coefficient with depth under the sediment-water interface and is here considered as formed by 6 layers, each characterized by a constant diffusion coefficient.

A global sensitivity analysis is performed for Case 2 to evaluate the relative importance of model parameters on diffusive transport and mixing. Note that concentration values are normalized by the maximum initial concentration and are given in $[\%]$.

\section{A. Case 1: Bimaterial medium}

We consider a bimaterial medium of finite extent and characterized by a discontinuity in the diffusion coefficient at the interface between two materials. Focusing on this exemplary setting enables us to $(a)$ benchmark the SBM model ${ }^{34}$ for solute transport under a discontinuous diffusion coefficient and (b) assessing the impact of the coupling between PSP and SBM on layer-averaged concentration and mixing in a simple case. We rely on dimensionless quantities and pattern the system according to the benchmark test presented by Lejay and Pichot $^{34}$. The latter comprises two layers, i.e., the Upper layer (associated with vertical coordinates $0<y<3$ ) and the Lower layer $(-3<y<0)$, the discontinuity (interface) being located at $y_{I}=0$ (see Figure 2).

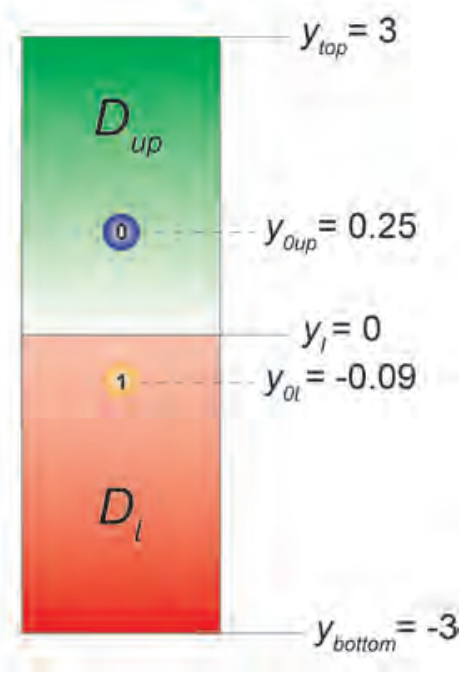

FIG. 2. Domain Case 1

The diffusion coefficients (termed $D_{u p}$ and $D_{l}$ for the $U p$ per and Lower layer, respectively) are piece-wise constant. As 
an initial condition, particles with a scalar value $\phi=1$ are located in the Lower layer at $y_{0_{l}}=-0.09$.

We start by validating our implementation of the SBM mode $^{28,34}$ for the evaluation of the one-dimensional diffusive transport scenario in the computational domain depicted in Figure 2. For this aim, the PSP model is not active and mixing is therefore neglected. The parameters used in the benchmark test are listed in Table I.

TABLE I. Parameters associated with the benchmark test used for validation of the SBM model: $D$ (Diffusion coefficient), $N_{p}$ (Numbe of particles), $y_{I}$ (Interface position), $\delta t_{t}$ (Time step), and $n_{t}$ (Number of time steps)

\begin{tabular}{ccc}
\hline \hline & Upper layer & Lower layer \\
\hline & & \\
$D$ & $D_{u p}=2$ & $D_{l}=5$ \\
$N_{p}$ & 100000 & 100000 \\
\multicolumn{4}{c}{ Simulation parameters } \\
\hline$=\frac{D_{l}}{D_{u p}}$ & 2.5 \\
$y_{I}$ & 0 & \\
$\delta t_{t}$ & 0.01 \\
$n_{t}$ & 30 \\
\hline \hline
\end{tabular}

The PDF of particle positions $q\left(t, y_{0}, y\right)$ at each time step (and hence particle concentration at a given location $y_{i}(t)$ ) is evaluated for both particles with the initial location at $y_{0_{l}}$ and $y_{0_{u p}}$ through the analytical expression ${ }^{34}$

$$
q\left(t, y_{0}, y\right)=\frac{1}{\sqrt{2 D(y)}} p_{\theta}\left(t, \frac{y_{0}}{\sqrt{2 D\left(y_{0}\right)}}, \frac{y}{\sqrt{2 D(y)}}\right)
$$

where $D\left(y_{0}\right)$ is the diffusion coefficient associated with the portion of the domain corresponding to the initial position of the particles, $D(y)$ being its counterpart in the region where a given particle is found at time $t$.

The density transition function $\left(p_{\theta}\right)$ of the Skew Brownian motion is expressed as ${ }^{33,34}$

$$
p_{\theta}\left(t, y_{0}, y\right)=\mathfrak{g}\left(t, y-y_{0}\right)+\operatorname{sgn}(y) \theta \mathfrak{g}\left(t,|y|+\left|y_{0}\right|\right)
$$

where,

$$
\text { sgn }= \begin{cases}>0 & \text { if } y-y_{I}>0, \\ <0 & \text { if } y-y_{I}<0,\end{cases}
$$

and the function $\mathfrak{g}\left(t, y-y_{0}\right)$ is defined as

$$
\mathfrak{g}\left(t, y-y_{0}\right)=\frac{1}{\sqrt{2 \pi t}} e^{-\frac{\left(y_{0}-y\right)^{2}}{2 t}}
$$

Numerical results are in close agreement with the analytical solution, thus imbuing us with confidence about the successful validation of our implemented SBM numerical code (see Figure 3(a)-(b)). The slight discrepancy observed between the analytical and the numerical results at $t=0.30$ is related to the observation that, while we assume that the particles are reflected at $y= \pm 3$, the analytical solution in Eq. (20) is associated with an infinite domain. As an additional example, Figure 3(c) depicts the root mean squared error (RMSE) between the analytical and the numerical density transition function at a selected time (i.e., $t=0.10$ ) versus the number of particles employed in the numerical simulation. The expected Monte Carlo convergence rate is observed, with $\mathrm{RMSE} \propto N_{p}^{-0.5}$.

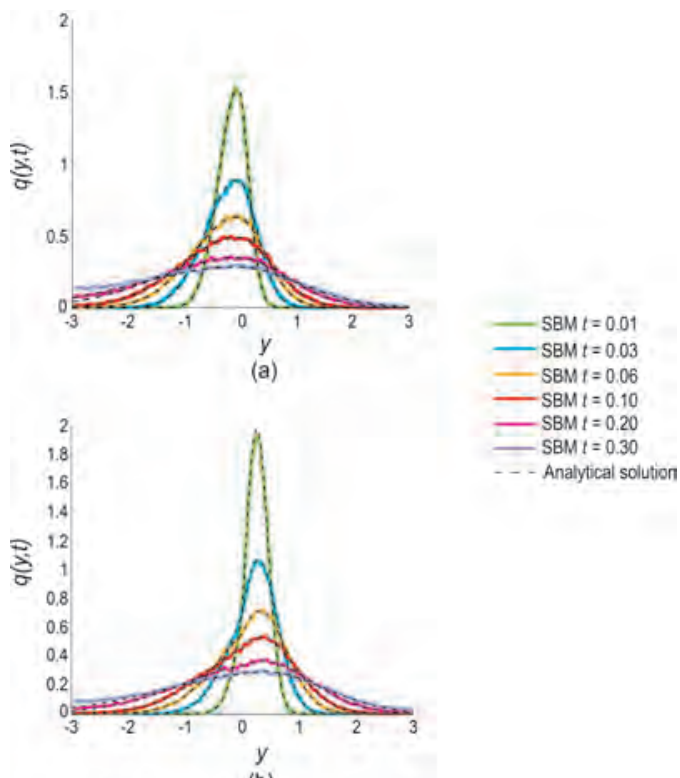

(b)

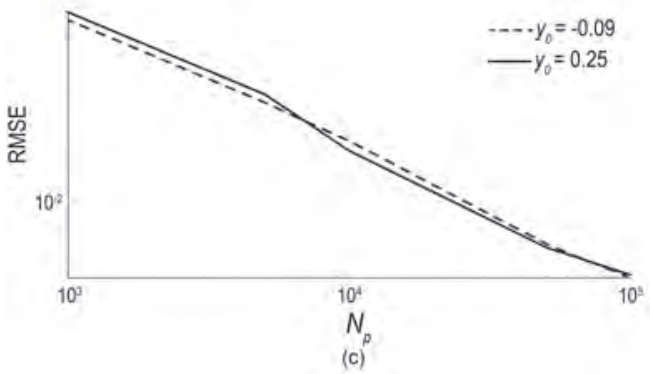

FIG. 3. $(a-b)$ Comparison between the numerical (thick colored curves) and the analytical (black dashed curves) density transition function $q\left(t, y_{0}, y\right)$ evaluated at selected time steps $(0.01 \leq t \leq 0.3)$ and for two diverse initial locations: $y_{0 l}=-0.09(a)$ and $y_{0 u p}=0.25$ (b). Values of $(c)$ RMSE between the analytical and numerical values of $q\left(t, y, y_{0}\right)$ versus the number of particles used in the simulation at $t=0.10$.

We then consider the same test case, where mixing is as- 
sumed to take place solely between particles within the same layer. We analyze the impact of the variability (or uncertainty) of model parameters on selected outputs. We recall that each particle is marked by a different value of $\lambda_{i}$, which is randomly sampled from a uniform distribution defined within the support $\left[\lambda_{\min }, \lambda_{\max }\right]$ (Section II B). We consider the following four uncertain model parameters

- $\rho=\frac{D_{l}}{D_{u p}}$, corresponding to the ratio between the diffusion coefficient of the Lower layer $D_{l}$ and the Upper layer $D_{u p}$.

- $\frac{C_{t}}{C_{\phi}}$, i.e., the PSP model parameter ${ }^{29}$, as defined in Section II B;

- $\lambda_{\min }, \lambda_{\max }$, respectively corresponding to the lower and upper bound of the range of values which can be undertaken by $\lambda_{i}$.

Values of each of these parameters is sampled from a uniform distribution with a standard deviation equal to $20 \%$ of the corresponding mean. The mean values are taken from Lejay and Pichot $^{34}$ for $\rho$ or in Meyer ${ }^{29}$ for $\frac{C_{t}}{C_{\phi}^{\prime}}$. In our example,

Eq. (23) is employed upon setting $\bar{w}$ and $C_{\phi}^{\prime}$ at the values used in Meyer $^{29}$ and $D$ as the average of the values of the diffusion coefficients listed in Table I. We also perform a model evaluation upon setting the parameters to the above mentioned mean values of their corresponding ranges (see Table III). These results can be used as a reference to identify trends associated with model results and to allow a direct comparison of the solution obtained through SBM with and without coupling it with the PSP mixing model.

$$
\bar{\lambda}=\pi \sqrt{\frac{2 D}{C_{\phi}^{\prime} \bar{w}}}
$$

Lower and upper extremes of the supports of the considered uncertain model parameters are listed in Table II.

TABLE II. Extremes of the sampling range of the model parameter

\begin{tabular}{ccc}
\hline \hline & $\min$ & $\max$ \\
\hline & & \\
$\rho$ & 1.634 & 3.366 \\
$\frac{C_{t}}{C_{\phi}^{\prime}}$ & 0.0980 & 0.2019 \\
$\lambda_{\min }$ & 0.0980 & 0.2019 \\
$\lambda_{\max }$ & 0.2026 & 0.4173 \\
$\lambda$ & $\lambda_{\min }$ & $\lambda_{\max }$ \\
& & \\
\hline \hline
\end{tabular}

Layer-averaged solute concentrations resulting exclusively from transport (SBM) are compared against their counterparts obtained from the combined effect of mixing and transport (SBM + PSP).

Values of (spatially) averaged concentrations increase in time for the Upper layer and decreases for the Lower layer.
TABLE III. Values of the model parameters considered for the comparison between results stemming from SBM and SBM+PSP, as depicted in Figure 4

\begin{tabular}{cc}
\hline \hline & \\
$\rho$ & 2.5 \\
$D_{l}$ & 5 \\
$D_{u p}$ & 2 \\
$\frac{C_{t}}{C_{\phi}^{\prime}}$ & 0.15 \\
$\lambda_{\min }$ & 0.15 \\
$\lambda_{\max }$ & 0.31
\end{tabular}

We recall that a major requirement of any mixing model ${ }^{43}$ is that the mean concentration of the total system, as quantified by Eq. (15), does not vary in time. As an example of our results, Figure 4 depicts the temporal variation of the mean concentration in the Lower layer associated with $(a)$ the reference solutions obtained through SBM with and without coupling it with the PSP mixing model (continuous and dashed curves, respectively) and $(b)$ the collection of realizations obtained upon sampling the model parameter space. The corresponding values of mean concentration resulting from the analytical formulation (21) are also depicted. These results show a larger reduction of the solute concentration under the combined effect of the diffusive transport and mixing (SBM + PSP) with respect to the scenario characterized solely by transport processes (SBM), which is essentially overlapped with the analytical results. The shaded area in Figure 4(a) corresponds to the envelope of average solute concentration histories obtained for each parameter realization over a total number of 300 realizations. This result shows that the variability of the parameters is transferred onto the concentration values observed in the layer. At sufficiently long times, considering mixing leads to a decrease of concentration, as seen by the shaded area which lies generally below the dashed curve.

The temporal evolution of the volume-weighted (as estimated through Eq. (16)) concentration PDF across the whole domain is illustrated in Figure 5 for the reference realization characterized by the model parameter values listed in Table III.

The PDF of the concentration at time $t=0$ is a double delta function, as it corresponds to the initial configuration consisting of particles with scalar values equal to 0 and 1 at the Upper and Lower layer, respectively. As a consequence of mixing, the PDF evolves to a final configuration displaying a bimodal character, with peaks at $C \approx 40 \%$ and $C \approx 60 \%$ (these values corresponding to the layer averaged concentrations at the $U p$ per and Lower layer, respectively) and whose average is equal to the mean concentration across the whole system. Due to the spatial heterogeneity in the diffusion coefficient, the distribution displays a mild skewness at intermediate times.

Mixing in the whole domain is assessed through the analysis of $C^{2}$ and $\chi$. A time filter is applied to compute the scalar dissipation rate through numerical approximation of Eq. (19) to reduce the noise and improve the quality of visualization of results. Figure $6(a)$ shows that $\bar{C}^{2}$ tends to decrease over time, 


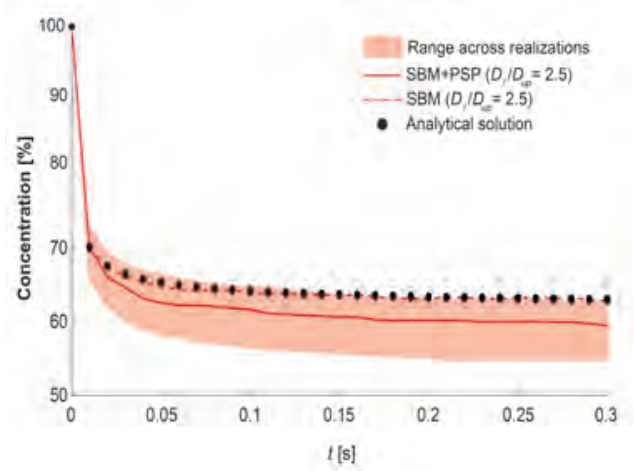

FIG. 4. Comparison of the average solute concentration at the Lower layer resulting from SBM (red dashed curve) and the combination of SBM and PSP (red solid curve), obtained by setting model parameters as in Table III. Results based on the analytical solution corresponding to Eq. (20) are also included (black dotted curves). Shaded areas correspond to the envelope of concentration histories associated with 300 realizations of model parameters sampled across the intervals listed in Table II.

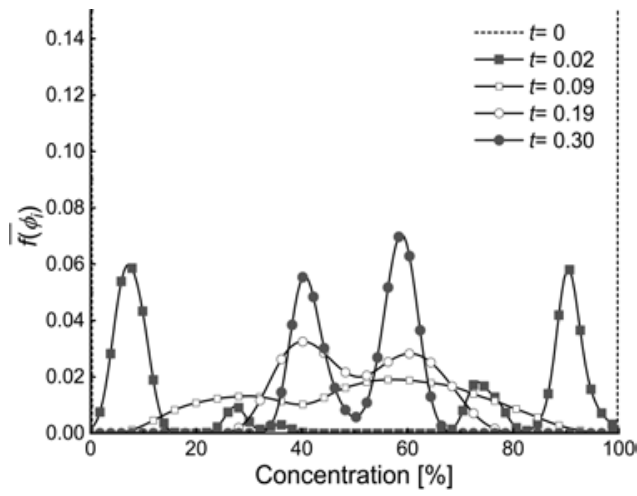

FIG. 5. Case 1: Volume-weighted (see Eq. (16)) probability density function of the solute concentration $\bar{f}\left(\phi_{i}\right)$ obtained with parameters listed in Table III.

showing the steepest gradient values until time level $t=0.15$ and then tending towards an asymptotic value, corresponding to a situation where particles are uniformly mixed across the system. The decrease of the scalar dissipation rate displayed in Figure $6(b)$ suggests that the mixing rate is highest at the early stages $(t<0.05)$ and decreases with a constant gradient until $t=0.15$.

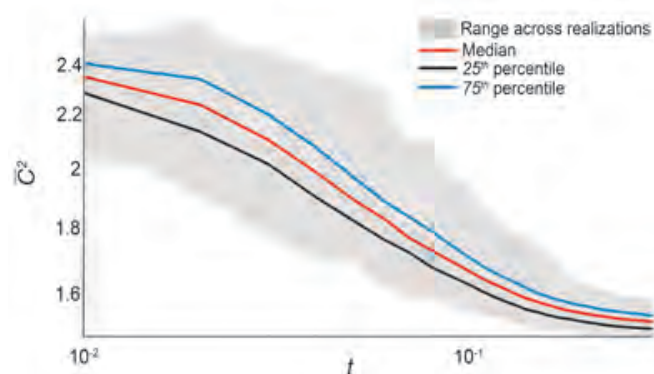

(a)

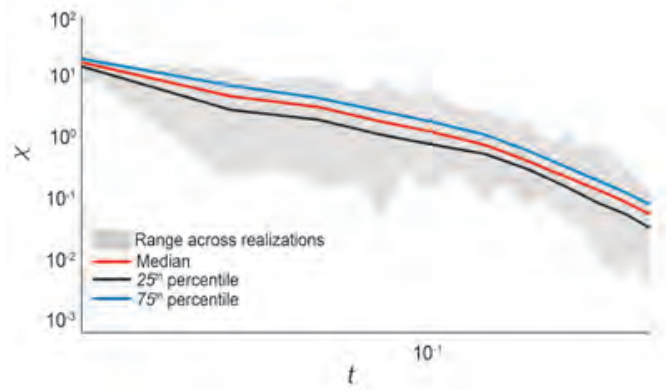

(b)

FIG. 6. Case 1: Temporal variation of $\overline{C^{2}}(a)$ and $(b)$ median (red line), $25^{\text {th }}$ percentile (black line), and $75^{\text {th }}$ (blue line) percentile of the scalar dissipation rate. The grey shaded regions encompass the collection of realizations of $C^{2}$ and $\chi$ values.

\section{B. Case 2: Erosimeter}

The second scenario we analyze consideres the simulation of transport and mixing within a system mimicking the experimental setup (erosimeter) adopted in Chandler et al. ${ }^{10}$ and corresponding to a porous medium underlying a water column. A sketch of the system is depicted in Figure 7.

The porous domain is constituted by grains with uniform diameter $d_{g}=0.005 \mathrm{~m}$ and a porosity $\Phi=0.39$. In the experimental setup considered by Chandler et al. ${ }^{10}$, turbulence is generated at the top of the sediment-water interface by a propeller producing a bed shear velocity $u=0.01 \frac{\mathrm{m}}{\mathrm{s}}$ at the exchange interface. Our aim is to simulate vertical solute exchange taking place in the system as driven by diffusion and mixing. Experimental ${ }^{10}$ and numerical ${ }^{42}$ results document an exponential reduction of the diffusion coefficient with increasing depth below the sediment-water interface. For the purpose of our study, the porous domain is then segmented into 6 layers (see Figure 7). As initial configuration, particles are distributed proportionally to the fluid volume present in each layer, mass and concentration values being set in a way that a 


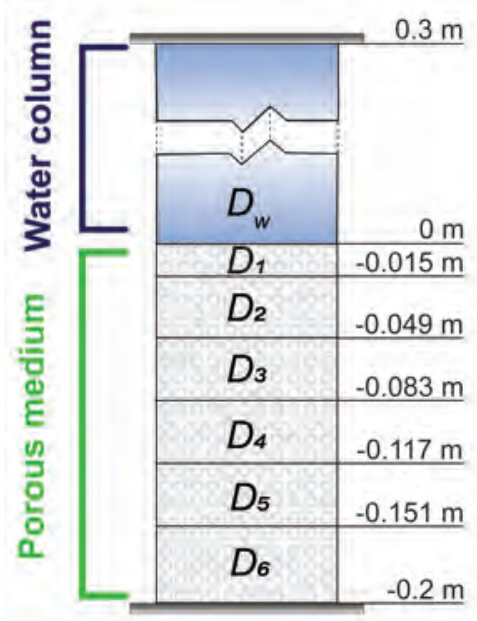

FIG. 7. Sketch of the domain for Case 2 mimicking an erosimeter

spatially uniform unit concentration is attained in the porous medium, while solute concentration is zero in the overlying water column. We illustrate two separate sets of results. First, we quantify the impact of the spatial variability of the diffusion coefficient on mixing within the porous medium. We then perform a sensitivity analysis to assess the relative importance of model parameters on the observed behaviors.

\section{Mixing under spatially discontinuous diffusivity}

We first investigate how the considered spatial heterogeneity of the diffusion coefficient affects solute mixing. Two scenarios are examined

1. Scenario 1: Variable diffusion coefficient: The diffusion coefficient $D_{k}$ is considered as a constant within each layer $k$ while being characterized by a vertical variability through the following equation

$$
D_{k}(y)=A e^{B y_{k}}
$$

where $A$ and $B$ are model parameters, and $y_{k}$ is the distance between the center of layer $k$ and the sedimentwater interface.

2. Scenario 2: Constant D: The diffusion coefficient $D_{k}$ is constant across the entire porous domain, its value being estimated as a weighted average of the diffusion coefficient values associated with Scenario (1) according to

$$
\bar{D}=\frac{\sum_{k=1}^{N_{L p}} D_{k} V_{k}}{\sum_{k=1}^{N_{L p}} V_{k}}
$$

Input values considered for model parameters are listed in Table IV. The profile length scale is estimated upon relying on available experimental data ${ }^{10}$. For Scenario (1), the SBM stage is repeated 10 times with a time step $\delta t_{t}=\frac{1}{10}$ of the corresponding time step $\left(\delta t_{m}\right)$ associated with mixing to avoid the occurrence of large displacements within a given time step due to the effect of diffusion at locations close to the sedimentwater interface. In this context, we recall that, as suggested in Lejay and $\mathrm{Pichot}^{34}$, an appropriate choice of $\delta t_{t}$ is pivotal to ensure an accurate depiction of particle's behavior, especially close to a discontinuity (i.e., the interface between layers). Both $\delta t_{t}$ and $\delta t_{m}$ are assumed equal to $1 s$ for Scenario (2), which yielded acceptable accuracy in preliminary tests.

TABLE IV. Values used for the key model variables for the comparison between numerical results associated with Scenario (1) and Scenario (2): Number of particles $\left(N_{p}\right)$, initial time $\left(t_{0}\right)$, number of time steps $\left(n_{t}\right)$, time step of SBM $\left(\delta t_{t}\right)$, time step associated with mixing $\left(\delta t_{m}\right)$, porosity $(\Phi)$, diffusion coefficient of the water column $\left(D_{w}\right)$, diffusion coefficient within the porous medium Scenario (2) $(\bar{D})$, and model parameters $A, B, \frac{C_{t}}{C_{\phi}^{\prime}}, \lambda_{\min }, \lambda_{\max }$

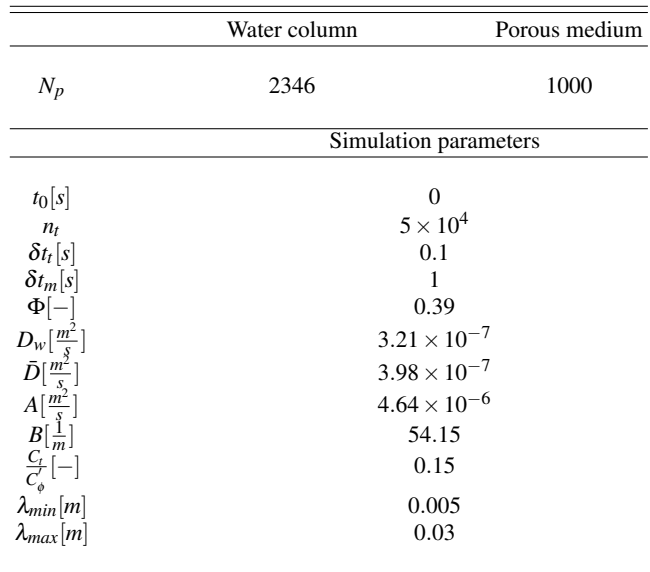

Figure 8 depicts the comparison between the two scenarios in terms of $\bar{C}^{2}$ and scalar dissipation rate, evaluated through Eq. (17) and Eq. (19), respectively. A quicker reduction of $\bar{C}^{2}$ is observed in Figure $8(a)$ at early stages $\left(t<2 \times 10^{4} \mathrm{~s}\right)$ when a discontinuous diffusion coefficient is assumed (Scenario 1). At first, the temporal decrease of $\bar{C}^{2}$ takes place at similar rates in both scenarios, with a slightly larger rate for the variable diffusion setting. This result suggests that mixing between the water column and the shallow layers of the porous space occurs faster in Scenario (1) as compared 
against Scenario (2) due to a higher difference between the diffusion coefficient in the water column and Layer 1. Otherwise, the opposite behavior is observed at later times (i.e. $t>5 \times 10^{3} \mathrm{~s}$ ), the temporal reduction rate of $\bar{C}^{2}$ being larger in Scenario (2) than in Scenario (1). When mixing between the regions across the exchange interface attains equilibrium (i.e., mixing starts to affect the lowest layers), $\bar{C}^{2}$ in Scenario (2) reduces more slowly than in Scenario (1). These results are then reflected by the time evolution of scalar dissipation rate in Figure 8(b). The predicted evolution of $\chi$ is compared to the trend $\chi \propto t^{-3 / 2}$ associated with a one-dimensional diffusive regime ${ }^{44}$ characterized by a constant diffusion coefficient. We observe that $\chi$ in Scenario (1) denotes a trend which is closer to the reference $t^{-3 / 2}$, while displaying a faster decay for Scenario (2), which is likely linked to enhanced mixing in the porous medium.

On the bases of the results illustrated above, we can conclude that the spatial variability of the effective diffusion coefficient tends to markedly affect mixing dynamics. This may in turn have strong consequences on the model-based quantification of mixing-driven reactive processes.

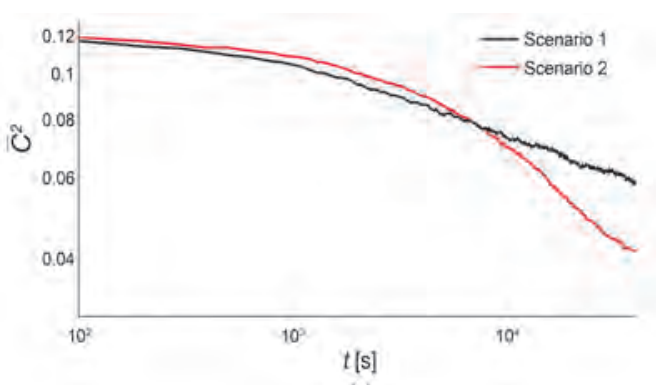

(a)

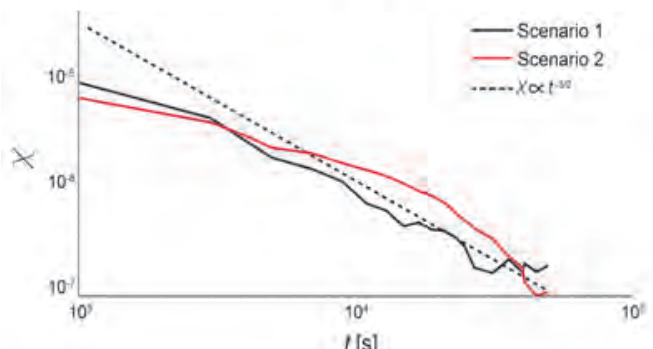

(b)

FIG. 8. Temporal variation of $\bar{C}^{2}(a)$ and of the scalar dissipation rate $\chi(b)$ for Scenario (1) and Scenario (2). The dashed line in $(b)$ corresponds to $\chi \propto t^{-3 / 2}$

\section{Sensitivity analysis}

A sensitivity analysis is performed to estimate the impact of model parameters on solute concentration and mixing (quantified through $C^{2}$ and $\chi$, respectively) in the presence of a spatially variable diffusion coefficients (i.e., Scenario 1). Parameters we consider in the sensitivity analysis are: $(i)$ the diffusion coefficient of the water column, $D_{w} ;(i i)$ parameters $A$ and $B$ driving the exponential decay of the diffusion coefficient within the porous medium according to Eq. (24); and (iii) three parameters of the PSP mixing model, i.e., $\frac{C_{t}}{C_{\phi}^{\prime}}, \lambda_{\text {min }}$ and $\lambda_{\max }$. These parameters are considered as independent and identically distributed random variables, each characterized by a uniform distribution within selected intervals, chosen considering a coefficient of variation of 0.2 . The mean values of the parameters $A$ and $B$ are determined upon relying on the results of Baioni et al. ${ }^{42}$, while average values of $\lambda_{\text {min }}$ and $\lambda_{\max }$ are set on the basis of existing experimental data ${ }^{10}$. The mean value of $\frac{C_{t}}{C^{\prime}}$ is set to the value employed in Meyer ${ }^{29}$. The parameters used in the simulation are listed in Table V.

TABLE V. Lower and upper bounds of the sampling intervals of model parameter values for the simulation of Scenario (1).

\begin{tabular}{|c|c|c|}
\hline & Water column & Porous medium \\
\hline$N_{p}$ & 2346 & 1000 \\
\hline & \multicolumn{2}{|c|}{ Simulation parameters } \\
\hline $\begin{array}{c}t_{0}[s] \\
\Phi\end{array}$ & \multicolumn{2}{|c|}{$\begin{array}{c}0 \\
0.39\end{array}$} \\
\hline & $\min$ & $\max$ \\
\hline$D_{w}\left[\frac{m^{2}}{s}\right]$ & $2.101 \times 10^{-7}$ & $4.327 \times 10^{-7}$ \\
\hline$A\left[\frac{m^{2}}{s}\right]$ & $3.035 \times 10^{-6}$ & $6.252 \times 10^{-6}$ \\
\hline$B\left[\frac{1}{m}\right]$ & 35.39 & 72.91 \\
\hline$\frac{C_{t}^{L m}}{C_{d}^{\prime}}[-]$ & 0.0980 & 0.2019 \\
\hline$\lambda_{\min }[m]$ & 0.0032 & 0.0067 \\
\hline$\lambda_{\max }[m]$ & 0.0196 & 0.040 \\
\hline$\lambda[m]$ & $\lambda_{\min }$ & $\lambda_{\max }$ \\
\hline
\end{tabular}

Time step values for the diffusive transport $\delta t_{t}$ and mixing $\delta t_{m}$ coincide with those used in Scenario (1). We first consider the temporal dynamics of $C_{3}$, i.e., the layer-averaged concentration of Layer 3 located in a central position of the considered porous domain (see Figure 9).

The average concentration reduces over time from the initial value of $C=100 \%$ displaying the highest decay rates at early stages. Concentration at Layer 3 is computed for various combinations of model parameters. The median over all realizations obtained by sampling the parameter space is evaluated for $(i)$ diffusive transport (i.e., SBM), and (ii) the combination of transport and mixing (i.e., SBM+PSP) (see Figure 

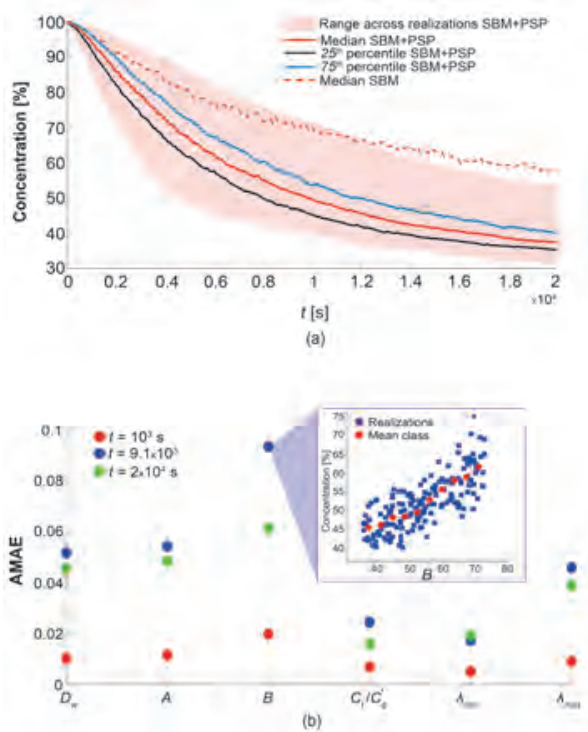

FIG. 9. Temporal evolution of the solute concentration in Layer $3 C_{3}$ considering only transport (SBM) and the combination of transport and mixing (SBM+PSP) $(a)$ : median value (SBM: red dashed curve; SBM +PSP: red solid curve); $25^{\text {th }}$ percentile SBM +PSP (black curve), $75^{\text {th }}$ percentile SBM +PSP (blue curve). The red shaded area represents the envelope of concentration values obtained for each realization under the combined effect of diffusive transport and mixing. Panel $(b)$ represents the AMAE sensitivity index defined in Eq. (26) for solute concentration at $t=10^{3} \mathrm{~s}, t=9.1 \times 10^{3} \mathrm{~s}$ and $t=2 \times 10^{4}$ $\mathrm{s}$. The red dotted curve is the average value of $C_{3}$ upon conditioning on given values of the parameter $B$ at time $t=9.1 \times 10^{3} \mathrm{~s}$

$9(a)$ ). A faster reduction of solute concentration is observed in the presence of mixing (see red curve in Figure $9(a)$ ) as compared to its counterpart obtained only through the SBM model (red dashed line in Figure $9(a)$ ), the steepest decrease being documented at early stages $\left(t<10^{4} \mathrm{~s}\right)$. The behavior depicted in Figure 9 is consistent with the results obtained for the PDF of concentrations at Layer 3 depicted in Figure $10(a)$. The PDF evolves from a delta function corresponding to the (deterministic) initial state to the final configuration exhibiting a peak value at $C \approx 30 \%$. Values associated with $C=100 \%$ and $t>0 \mathrm{~s}$ are related to the presence of unmixed particles (i.e., particles with a unit scalar value) entering Layer 3 from the underlying portion of the porous domain (Layer 4, 5, and $6)$.

The rate of temporal reduction of concentration strongly depends on the particular combination of model parameter values, as suggested by the extent of the red shaded area in Figure $9(a)$, which encompasses the results obtained for $C_{3}(t)$ acros the whole collection of realizations considered. We then evaluate the $A M A E$ global sensitivity index to quantify the rela-

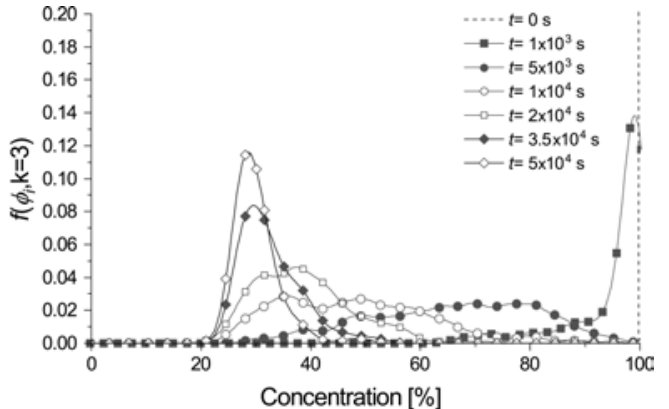

(a)

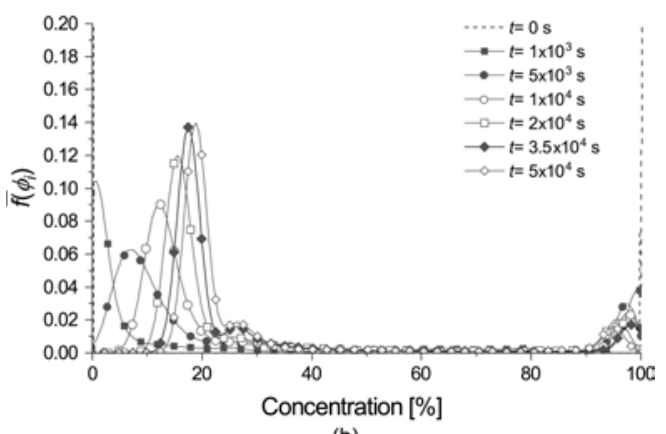

(b)

FIG. 10. Temporal evolution of the PDF of solute concentration for Layer $3(a)$ and volume weighted value across the whole domain evaluated $(b)$ upon setting model parameters to their mean value across their corresponding support.

tive influence of model parameters on the mean concentration at each layer. This metric was first introduced in Dell'Oca et al. ${ }^{45}$ with the aim of quantifying the effect of the uncertainty associated with a given model parameter $x_{i}$ on the expected value of a model output variable of interest, $y$, and is defined

$$
A M A E_{x_{i}}= \begin{cases}\frac{1}{\left|y_{0}\right|} E\left[\left|y_{0}-E\left[y \mid x_{i}\right]\right|\right], & \text { if } y_{0} \neq 0 \\ E\left[\left|y_{0}-E\left[y \mid x_{i}\right]\right|\right], & \text { if } y_{0}=0\end{cases}
$$

where $E[]$ denotes expected value, $E\left[y \mid x_{i}\right]$ denotes conditioning on $x_{i}$, and $y_{0}$ is the unconditional average of $y$, as a result of the uncertainty associated with the complete set of model parameters.

Figure $9(b)$ suggests that $B$ is the parameter with the highest effect on the mean concentration value. Considering a given observation time, Figure $9(b)$ clearly shows that the (conditional) average value of $C_{3}$ tends to increase with $B$. This is consistent with the observation that higher values of $B$ lead to lower diffusion coefficients within the porous domain and an ensuing slower decrease of solute concentration 
due to a reduced exchange between the water column and the shallowest layers. Moreover, we notice that the largest values of $A M A E$ are observed at $t=9.1 \times 10^{3} \mathrm{~s}$, lower values being attained as the mean concentration at Layer 3 approaches a constant value across parameter realizations. This temporal evolution of the mean value is also reflected in the variability observed in the local concentration values. Figure $10(b)$ also depicts the PDF of solute concentration across the whole domain evaluated upon setting model parameters to their mean value within their corresponding support. The PDF of solute concentration at Layer 3 evolves from a delta function corresponding to the initial configuration to a essentially Gaussian distribution with a mean at $C \approx 30 \%$. At intermediate stages, the PDF of concentration observed for the whole domain displays a markedly different behaviour. At time $t=0$ the PDF exhibits the shape of a double delta function corresponding to the initial configuration of $C=100 \%$ within the porous medium and $C=0 \%$ within the water column. The peaks at $C=100 \%$ and $C=0 \%$ tend to decrease over time due to the combined effect of transport and mixing. One can note that the sample probability of observing values of $C=100 \%$ is not zero even at the final stage of the process, suggesting that there are still unmixed particles in the domain at the lowest layers (Layer 5-6) at this late times. The peak of the distributions varies in time and attains a value of approximately $20 \%$ at time $t=5 \times 10^{4} \mathrm{~s}$. At this stage, the PDF is characterized by two peaks, corresponding to $C=20 \%$ and $C=100 \%$, respectively. The quantities $\bar{C}^{2}$ and $\chi$ are analyzed for various combinations of model parameter values sampled across their space of variability. Figure 11 $(a)$ shows a quicker decrease of $\bar{C}^{2}$ at early stages $\left(t<6 \times 10^{3}\right.$ s; i.e., when mixing acts across the water column and the shallowest layers) and a lower speed of reduction at the latest times (i.e., when mixing starts taking place also at the deepest locations). The scalar dissipation rate depicted in Figure 11 $(b)$ is obtained from $\bar{C}^{2}$ according to Eq. (19) and applying a time filter to avoid excessive noise in the results. We observe that the median values of $\chi$ tend to decay $\propto t^{-3 / 2}$ at long times. We also note that the decrease rate of $\chi$ appears to be realization-dependent, values of the scalar dissipation rate observed at the end of the simulation varying across more than one order of magnitude.

Figure 12 depicts the values of the $A M A E$ indices obtained for the scalar dissipation rate, thus quantifying the influence of each model parameter on the average mixing state. The highest value of the index is related to parameter $B$ at time $t=6 \times 10^{3} \mathrm{~s}$, an inverse proportionality between $B$ and the conditional expected value of $\chi$ being observed. We can note that all model parameters have essentially the same degree of importance (with similar values of the corresponding $A M A E$ indices) on the average mixing behavior at long times.

\section{CONCLUSIONS}

Our study explores the impact of the spatial variability of effective diffusion parameters on solute mixing. We leverage on two Lagrangian approaches to characterize transport and mixing of solute mass, i.e., the Skew Brownian Motion (SBM)

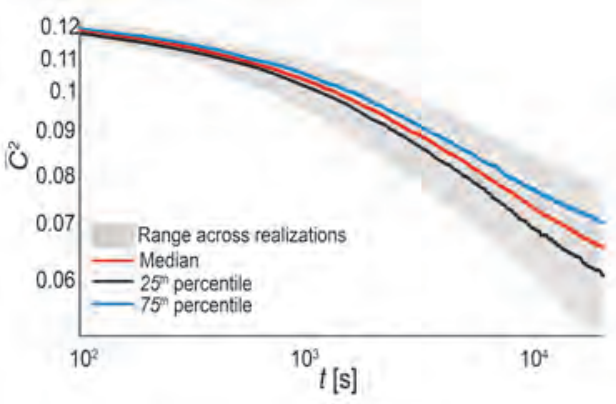

(a)

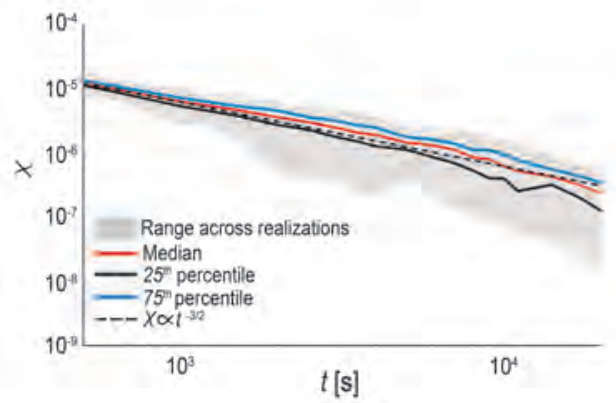

(b)

FIG. 11. Temporal variation of the median (red curve), $25^{\text {th }}$ (black curve), and $75^{\text {th }}$ (blue curve) percentile of $\bar{C}^{2}(a)$ and of the scalar dissipation rate $\chi(b)$. The grey shaded regions correspond to the envelope of all realization of $\bar{C}^{2}$ and $\chi$ values.

and the Parameterized Scalar Profile (PSP) models. These are applied to scenarios mimicking solute exchange in a hyporeic zone, i.e., close to a sediment-water interface, in the presence of turbulent flow. Our results lead to the following major conclusions.

- The implemented modeling workflow allows evaluating concentration and nonlinear indicators of mixing as well as the full probability distribution of concentration values. The resulting model is assessed through exemplary test cases, including the analysis of a scenario mimicking the experimental setup introduced by Chandler et al. ${ }^{10}$.

- When considering mass transport and mixing close to a sediment-water interface in the presence of turbulent flow, we observe that assuming an exponential decay of the diffusion coefficient with the depth below the interface between surface water and the porous domain has a marked influence on mixing dynamics, as opposed to a setting associated with a constant effective diffusivity 


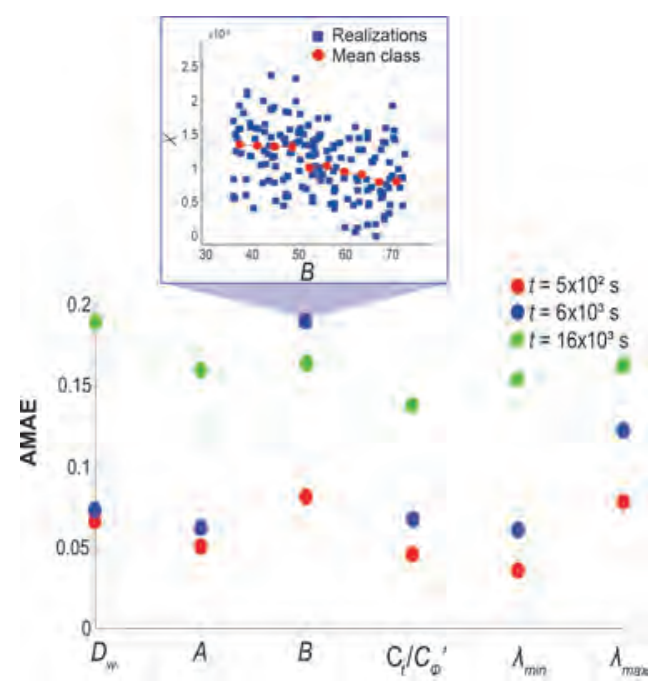

FIG. 12. AMAE index of the scalar dissipation rate $\chi$ for the whole domain at $t=5 \times 10^{2} \mathrm{~s}, t=6 \times 10^{3} \mathrm{~s}$ and $t=16 \times 10^{3} \mathrm{~s}$. The red dotted curve in the inset represents the average value of $\chi$ conditional to parameter $B$ at time $t=6 \times 10^{3} \mathrm{~s}$, blue squares denoting the values of $\chi$ as a function of $B$ for each realization sampled across the space of the remaining model parameters.

across the sediment volume.

- Since, to the best of our knowledge, this is the first time the PSP model is applied to porous media, we analyze the system response under parametric uncertainty. This enables us to diagnose the model response under a variety of conditions associated with multiple randomly selected combinations of model parameter values. The considered parametric uncertainty is propagated onto the scalar dissipation rate and its temporal history. Values of scalar dissipation rate may vary up to one order of magnitude within the collection of model realizations when considering a fixed observation time. This result suggests that the proposed model may be able to simulate of broad range of mixing rates when applied with differing parameters combinations, this being a desirable feature in terms of model flexibility. On average the scalar dissipation rate stemming from the combined action of transport and mixing processes displays a decreasing trend $\propto t^{-\frac{3}{2}}$ at long times. These results may constitute the basis for interpreting experimental investigations providing direct observation of solute mixing in a porous medium subject to turbulent flow.

Future work comprises $(i)$ an assessment of the illustrated modeling workflow against experimental data, including, e.g., (a) estimation of model parameter and the associated uncertainty as constrained against available data and $(b)$ propaga- tion of residual (i.e., after calibration) model parameter uncertainty onto modeling results to finally $(c)$ identify requirements for data acquisition campaigns and constrain uncertainty associated with our ability to describe mixing phenomena in the hyporheic region, and (ii) the extension of the analysis to reactive solute transport.

${ }^{1}$ S. Buss, Z. Cai, B. Cardenas, J. Fleckenstein, D. Hannah, K. Heppell, P. Hulme, T. Ibrahim, D. Kaeser, S. Krause, et al., "The hyporheic handbook: a handbook on the groundwater-surface water interface and hyporheic zone for environment managers," (2009).

${ }^{2}$ J. L. Schaper, M. Posselt, C. Bouchez, A. Jaeger, G. Nuetzmann, A. Putschew, G. Singer, and J. Lewandowski, "Fate of trace organic comA. Pulch pounds in the hyporhec zone. Influence of retardation, the benthic biolayer, and organic carbon," Environmental science \& technology 53, 4224-4234 (2019)

${ }^{3}$ J. Smith, "Groundwater surface water interactions in the hyporheic zone," Environment Agency , 1-209 (2005).

B. L. O'Connor and J. W. Harvey, "Scaling hyporheic exchange and its influence on biogeochemical reactions in aquatic ecosystems," Water Resources Research 44 (2008)

${ }^{5}$ J. M. Ottino and J. Ottino, The kinematics of mixing: stretching, chaos, and transport, Vol. 3 (Cambridge university press, 1989).

${ }^{6}$ P. d. Anna, J. Jimenez Martinez, H. Tabuteau, R. Turuban, T. Le Borgne, M. Derrien, and Y. Meheust, "Mixing and reaction kinetics in porous media: An experimental pore scale quantification," Environmental science technology 48, 508-516 (2014).

${ }^{7}$ M. Dentz, T. Le Borgne, A. Englert, and B. Bijeljic, "Mixing, spreading and reaction in heterogeneous media: A brief review," Journal of contaminant hydrology 120, 1-17 (2011).

${ }^{8}$ B. Jha, L. Cueto-Felgueroso, and R. Juanes, "Fluid mixing from viscous fingering," Physical review letters 106, 194502 (2011).

${ }^{9}$ T. Le Borgne, M. Dentz, and E. Villermaux, "The lamellar description of mixing in porous media," Journal of Fluid Mechanics 770, 458-498 (2015).

${ }^{10}$ I. Chandler, I. Guymer, J. Pearson, and R. Van Egmond, "Vertical variation of mixing within porous sediment beds below turbulent flows," Water resources research 52, 3493-3509 (2016).

${ }^{11}$ M. Higashino, J. J. Clark, and H. G. Stefan, "Pore water flow due to nearbed turbulence and associated solute transfer in a stream or lake sediment bed," Water resources research 45 (2009).

${ }^{12}$ M. J. de Lemos, "Turbulent kinetic energy distribution across the interface between a porous medium and a clear region," International communications in heat and mass transfer 32,107-115 (2005).

${ }^{13}$ K. Roche, A. Li, D. Bolster, G. Wagner, and A. Packman, "Effects of turbulent hyporheic mixing on reach-scale transport," Water Resources Research 55, 3780-3795 (2019)

${ }^{14}$ M. M. Nezhad, M. Rezania, and E. Baioni, "Transport in porous media with nonlinear flow condition," Transport in Porous Media 126, 5-22 (2019).

${ }^{15}$ C. Zhang, Y. She, Y. Hu, Z. Li, W. Wang, A. Patmonoaji, and T. Suekane, "Experimental investigation of solute transport in variably saturated porous media using X-ray computed tomography," Physics of Fluids 33, 076610 (2021)

${ }^{16}$ B. Ling, C. Rizzo, I. Battiato, and F. de Barros, "Macroscale transport in channel-matrix systems via integral transforms," Physical Review Fluids 6, 044501 (2021)

${ }^{17}$ J. S. Kim and P. K. Kang, "Anomalous transport through free-flow-porous media interface: Pore-scale simulation and predictive modeling," Advances in Water Resources 135, 103467 (2020).

${ }^{18}$ K. E. Bencala, "Simulation of solute transport in a mountain pool-and-riffle stream with a kinetic mass transfer model for sorption," Water Resources Research 19, 732-738 (1983).

${ }^{19}$ D. R. Hart, "Parameter estimation and stochastic interpretation of the transient storage model for solute transport in streams," Water Resources Research 31, 323-328 (1995).

${ }^{20}$ F. J. Triska, V. C. Kennedy, R. J. Avanzino, G. W. Zellweger, and K. E. Bencala, "Retention and transport of nutrients in a third-order stream in northwestern california: Hyporheic processes," Ecology 70, 1893-1905 (1989). ${ }^{21} \mathrm{~A}$. Wörman, "Comparison of models for transient storage of solutes in small streams," Water resources research 36, 455-468 (2000). 
${ }^{22}$ A. H. Elliott and N. H. Brooks, "Transfer of nonsorbing solutes to streambed with bed forms: Theory," Water Resources Research 33, 123 136 (1997).

${ }^{23}$ A. I. Packman and N. H. Brooks, "Hyporheic exchange of solutes and colloids with moving bed forms," Water Resources Research 37, 2591-2605 (2001).

${ }^{24}$ A. I. Packman, N. H. Brooks, and J. J. Morgan, "A physicochemical mode for colloid exchange between a stream and a sand streambed with bed forms," Water Resources Research 36, 2351-2361 (2000).

${ }^{25} \mathrm{~J}$. S. Fries, "Predicting interfacial diffusion coefficients for fluxes across the sediment-water interface," Journal of Hydraulic Engineering 133, 267-272 (2007)

${ }^{26}$ J. Y. Leung and S. Srinivasan, "Effects of reservoir heterogeneity on scaling of effective mass transfer coefficient for solute transport," Journal of contaminant hydrology 192, 181-193 (2016)

${ }^{27}$ F. J. Valdés-Parada, J. A. Ochoa-Tapia, and J. Alvarez-Ramirez, "Diffusive mass transport in the fluid-porous medium inter-region: Closure problem solution for the one-domain approach," Chemical Engineering Science 62, 6054-6068 (2007)

${ }^{28}$ A. Lejay and G. Pichot, "Simulating diffusion processes in discontinuous media: a numerical scheme with constant time steps," Journal of computational physics 231, 7299-7314 (2012)

${ }^{29} \mathrm{D}$. W. Meyer, On the modeling of molecular mixing in turbulent flows, Ph.D thesis, ETH Zurich (2008)

${ }^{30}$ C. Zhang, K. Kaito, Y. Hu, A. Patmonoaji, S. Matsushita, and T. Suekane, "Influence of stagnant zones on solute transport in heterogeneous porous media at the pore scale," Physics of Fluids 33, 036605 (2021).

${ }^{31} \mathrm{~K}$. Itô, P. Henry Jr, et al., Diffusion processes and their sample path (Springer Science \& Business Media, 2012).

${ }^{2}$ J. B. Walsh, "A diffusion with a discontinuous local time" Astérisque $\mathbf{5 2}$ 37-45 (1978).

${ }^{33}$ A. Lejay et al., "On the constructions of the skew brownian motion," Probability Surveys 3, 413-466 (2006).
${ }^{34}$ A. Lejay and G. Pichot, "Simulating diffusion processes in discontinuous media: Benchmark tests," Journal of Computational Physics 314, 384-413 (2016)

${ }^{35}$ D. W. Meyer and P. Jenny, "Micromixing models for turbulent flows," Journal of Computational Physics 228, 1275-1293 (2009).

${ }^{36} \mathrm{R}$. O. Fox, "The Fokker-Planck closure for turbulent molecular mixing: Passive scalars," Physics of Fluids A: Fluid Dynamics 4, 1230-1244 (1992)

${ }^{37} \mathrm{~N}$. Peters and P. Trouillet, "On the role of quasi-one-dimensional dissipation layers in turbulent scalar mixing," Annual Research Briefs , 27-40 (2002). ${ }^{38} \mathrm{~L}$. Wang and N. Peters, "The length-scale distribution function of the distance between extremal points in passive scalar turbulence," Journal of Fluid Mechanics 554, 457 (2006).

${ }^{39}$ S. B. Pope, "PDF methods for turbulent reactive flows," Progress in energy and combustion science 11, 119-192 (1985).

${ }^{40} \mathrm{R}$. O. Fox, Computational models for turbulent reacting flows (Cambridge university press, 2003)

${ }^{41}$ I. Chandler, Vertical variation in diffusion coefficient within sediments, Ph.D. thesis, University of Warwick (2012)

${ }^{42}$ E. Baioni, G. Porta, M. M. Nezhad, and A. Guadagnini, "Assessment of turbulence effects on effective solute diffusivity close to a sediment-free fluid interface," Stochastic Environmental Research and Risk Assessment 34, 2211-2228 (2020)

34, 2211-2228 (2020).
${ }^{43}$ S. Subramaniam and S. Pope, "A mixing model for turbulent reactive flows based on euclidean minimum spanning trees," Combustion and Flame 115 487-514 (1998)

${ }^{44}$ T. L. Borgne, M. Dentz, D. Bolster, J. Carrera, J. D. Dreuzy, and P. Davy, "Non-fickian mixing: Temporal evolution of the scalar dissipation rate in heterogeneous porous media," Advances in Water Resources 33, 1468 1475 (2010).

${ }^{45}$ A. Dell'Oca, M. Riva, and A. Guadagnini, "Moment-based metrics for global sensitivity analysis of hydrological systems," Hydrology and Earth System Sciences (2017). 

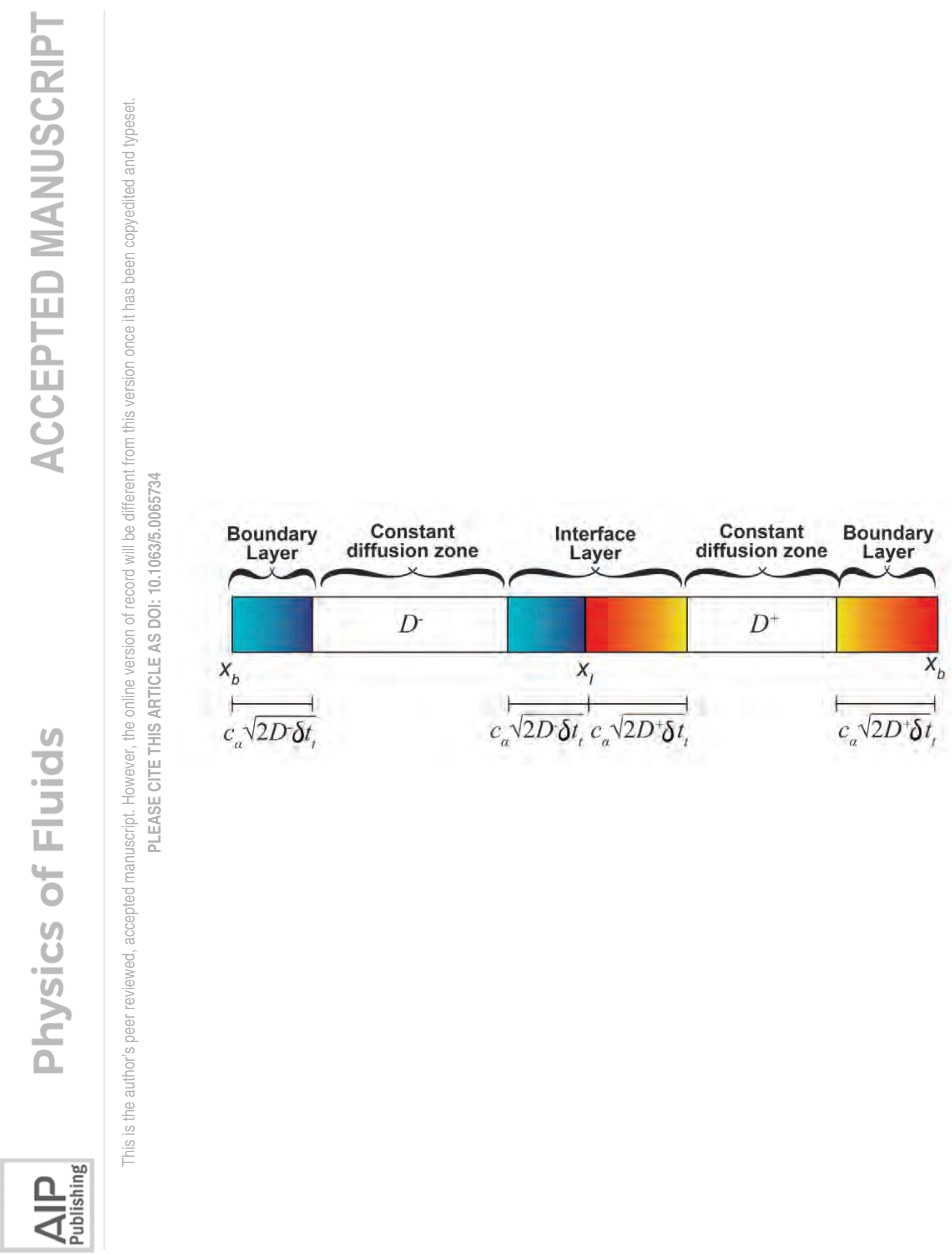

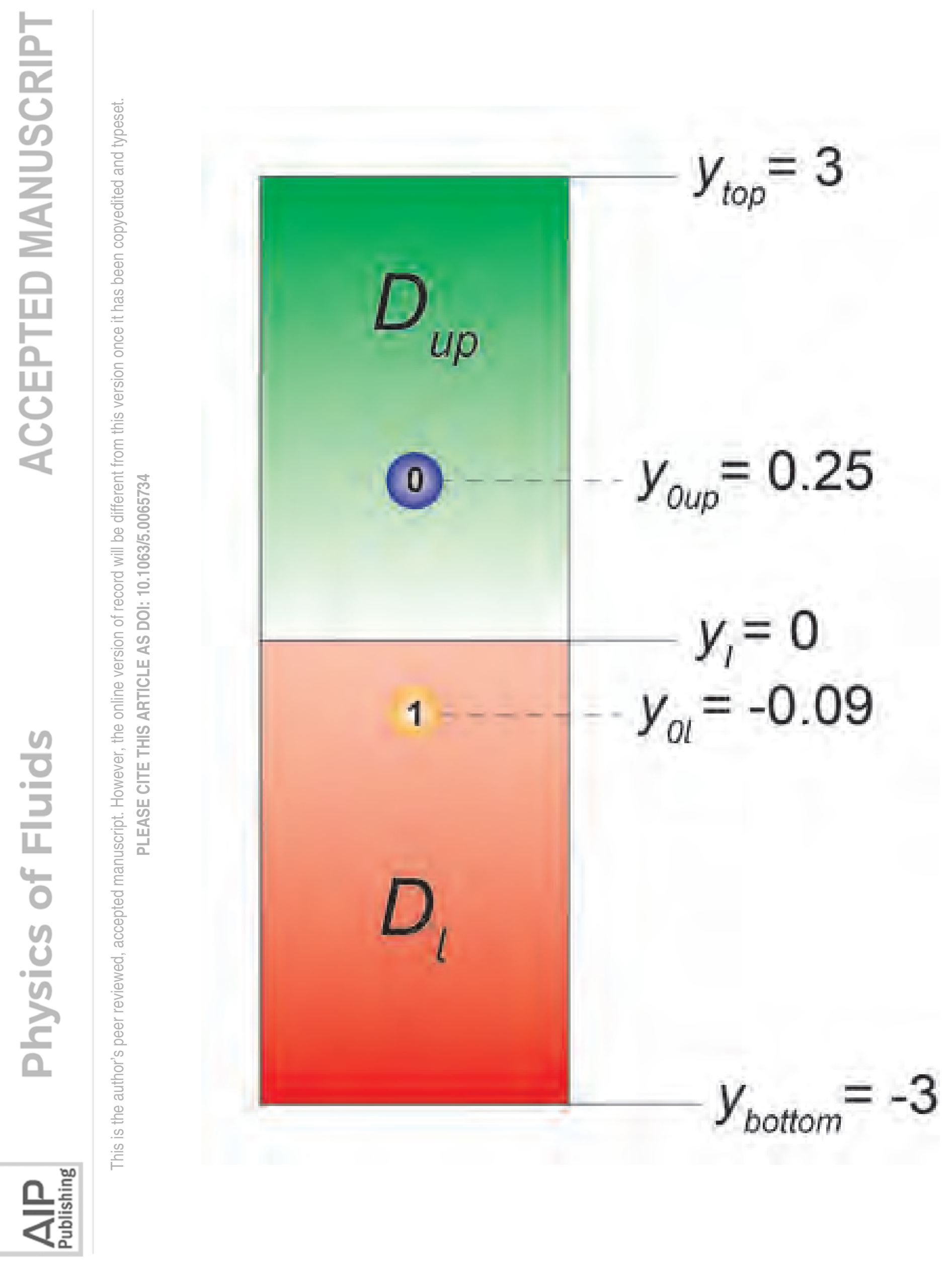


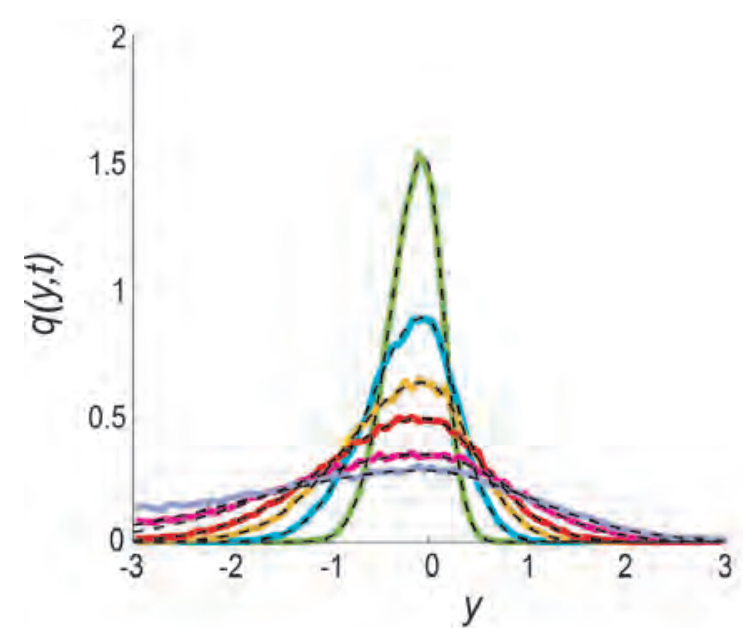

(a)

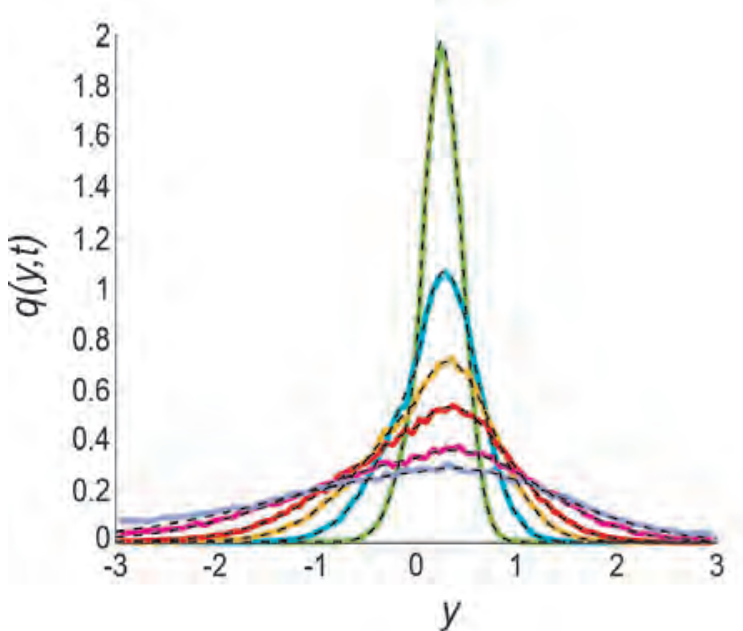

(b)

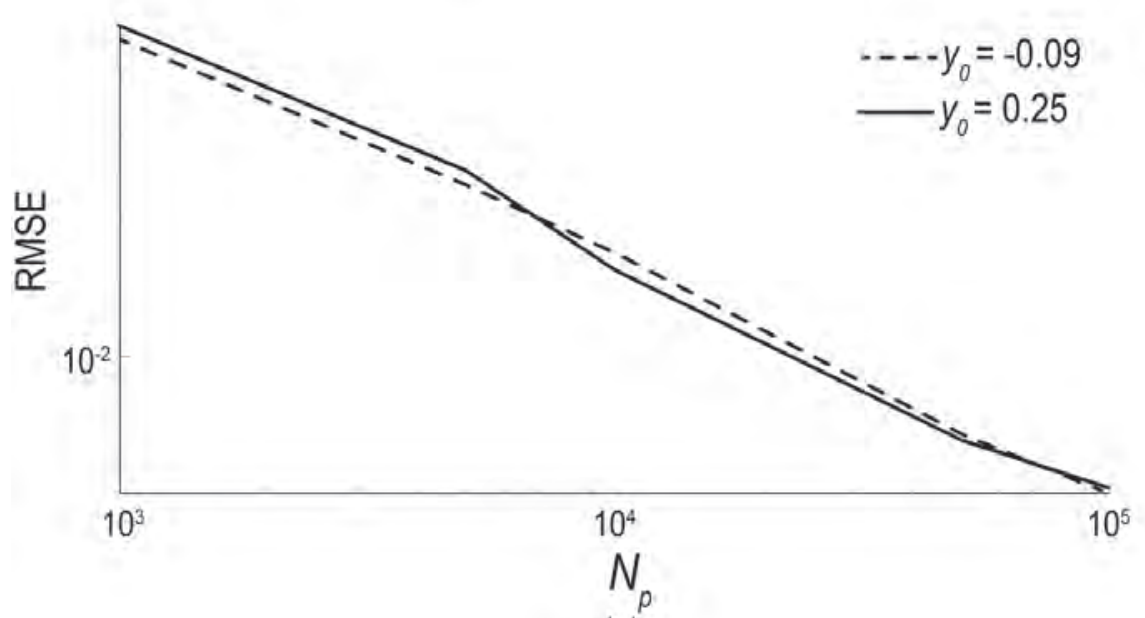

(c)

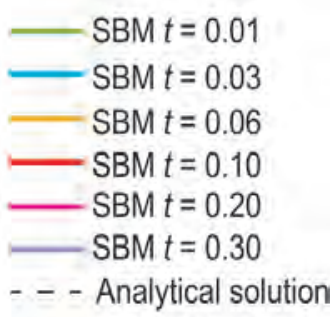

- - - Analytical solution

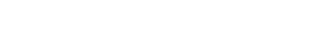




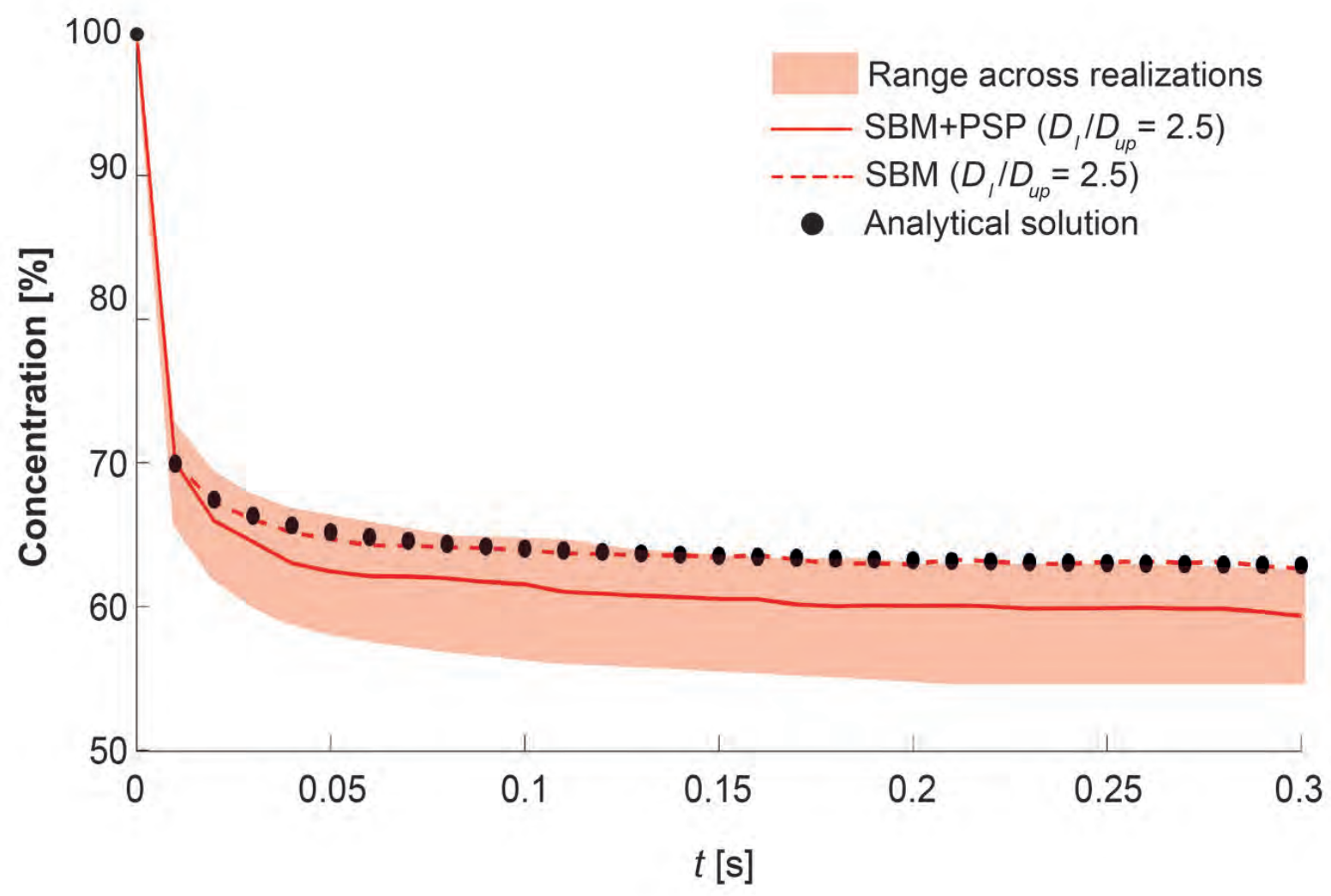




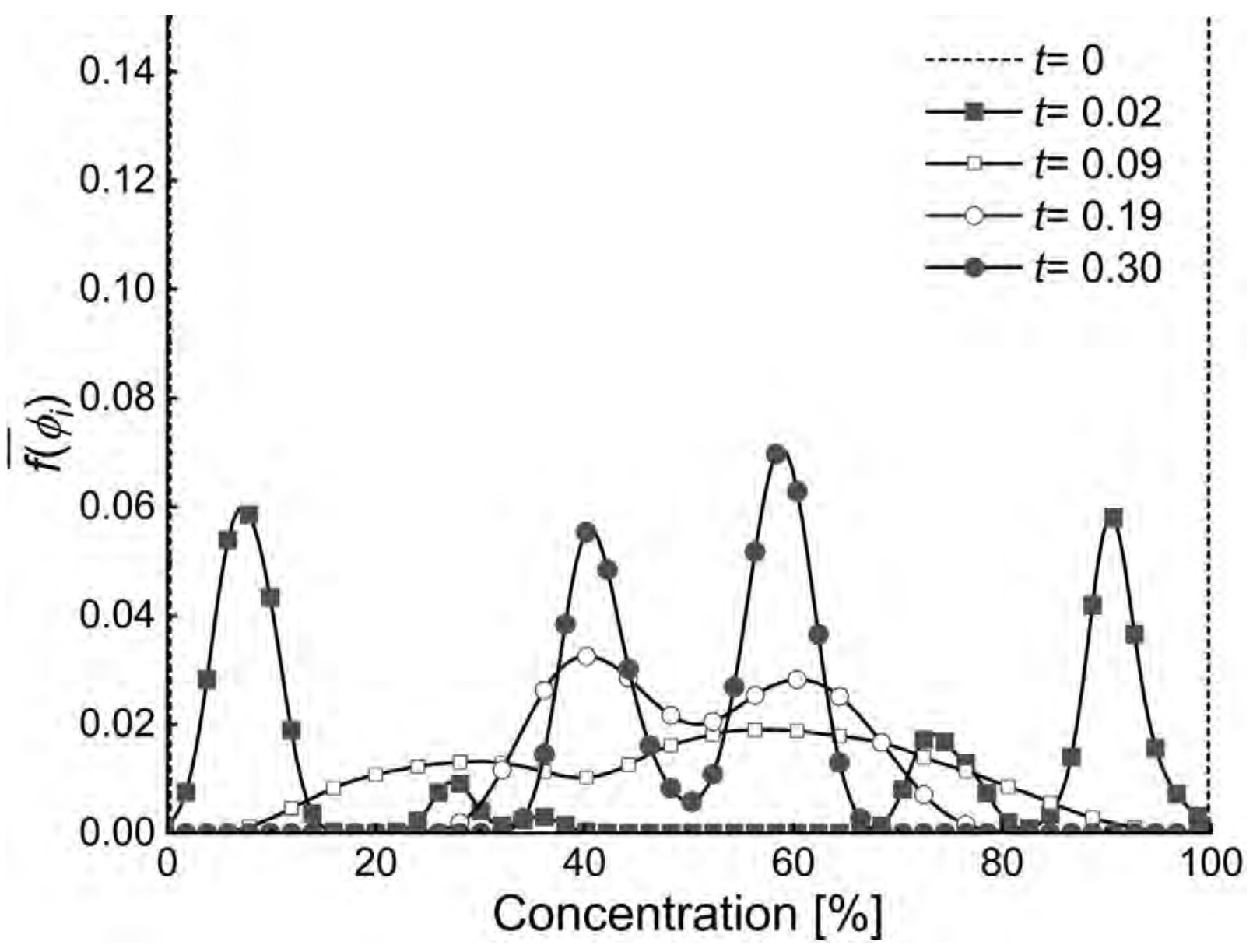

哭耪高 


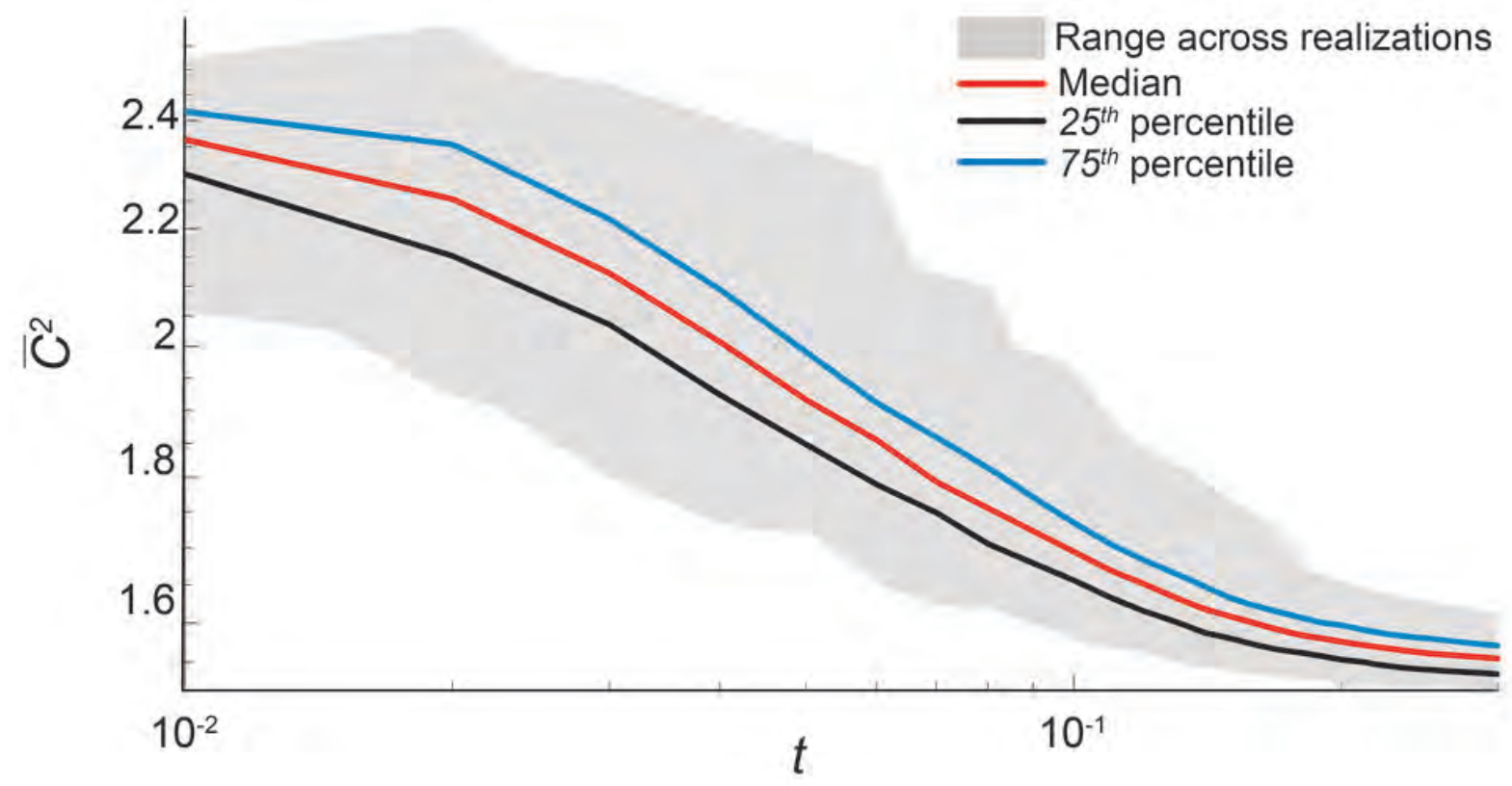

(a)

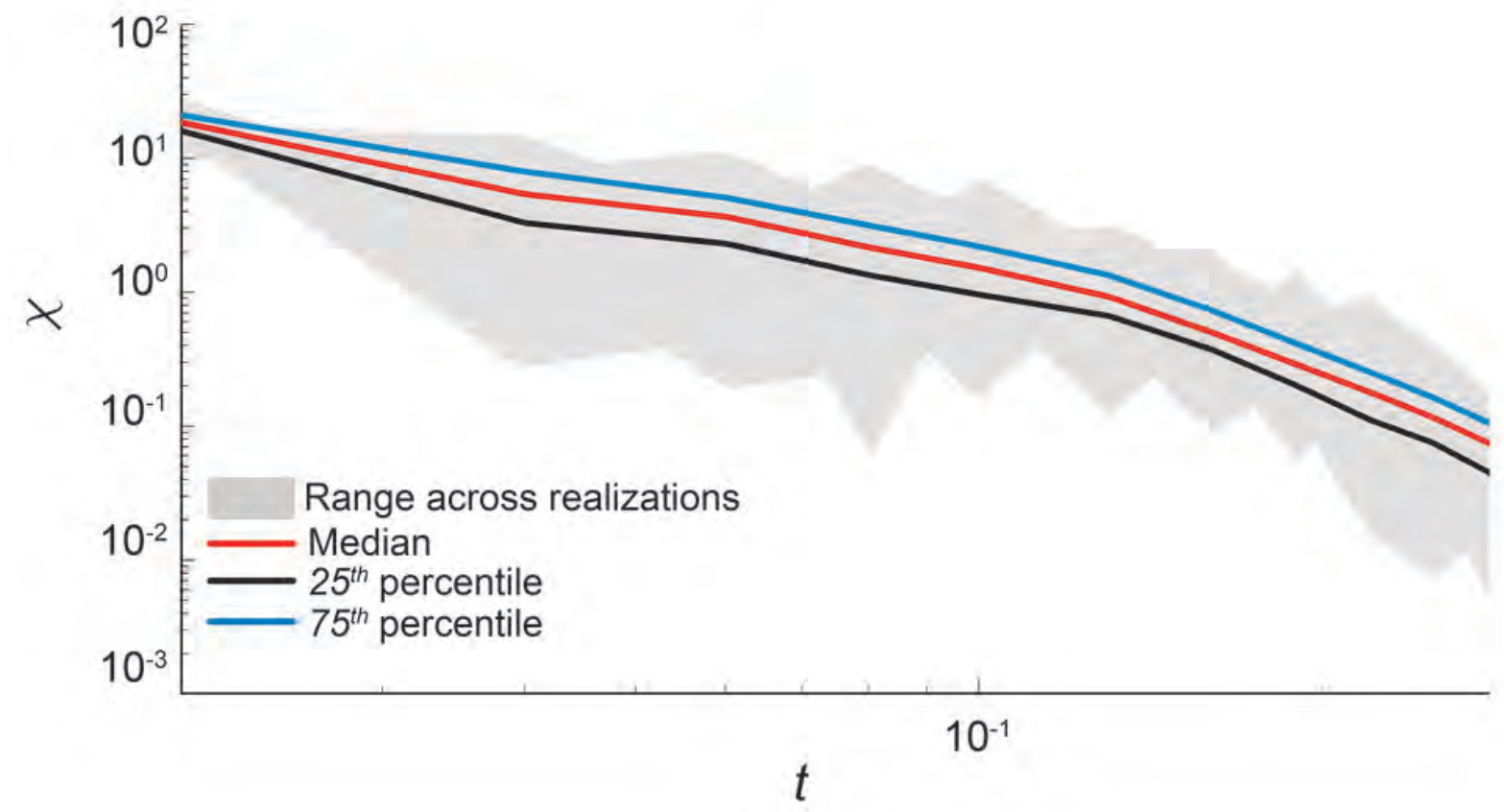

(b) 


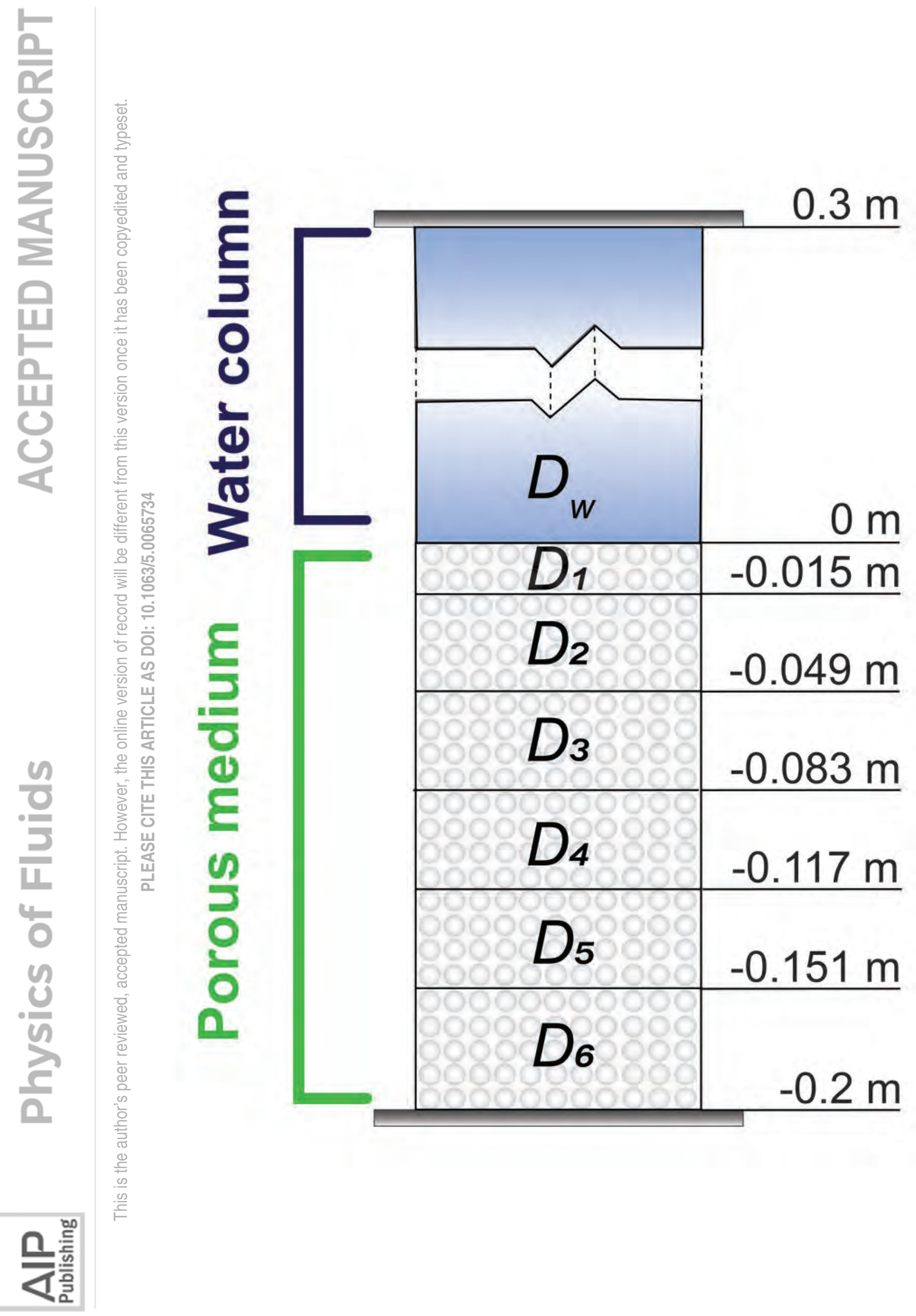




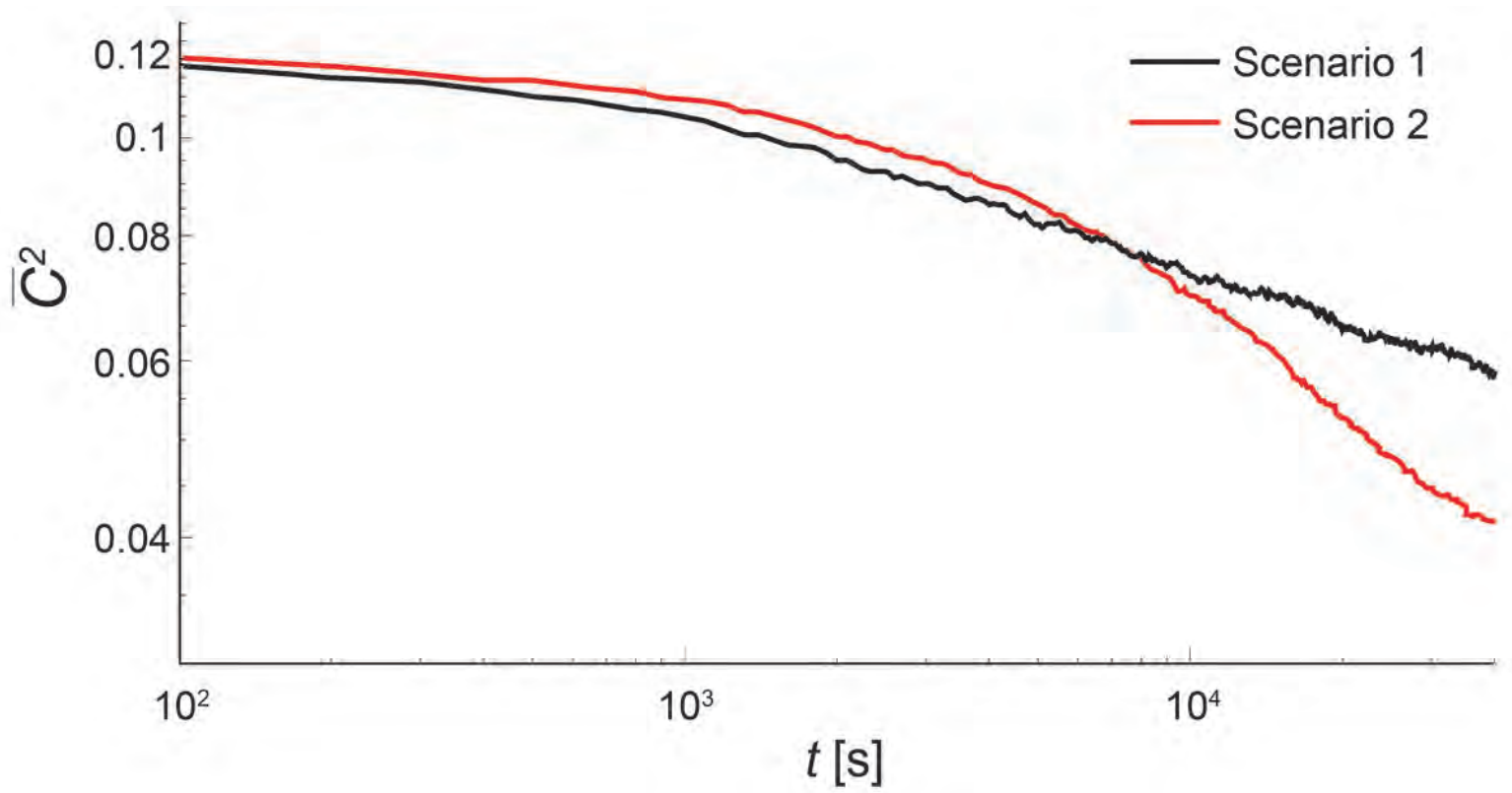

(a)

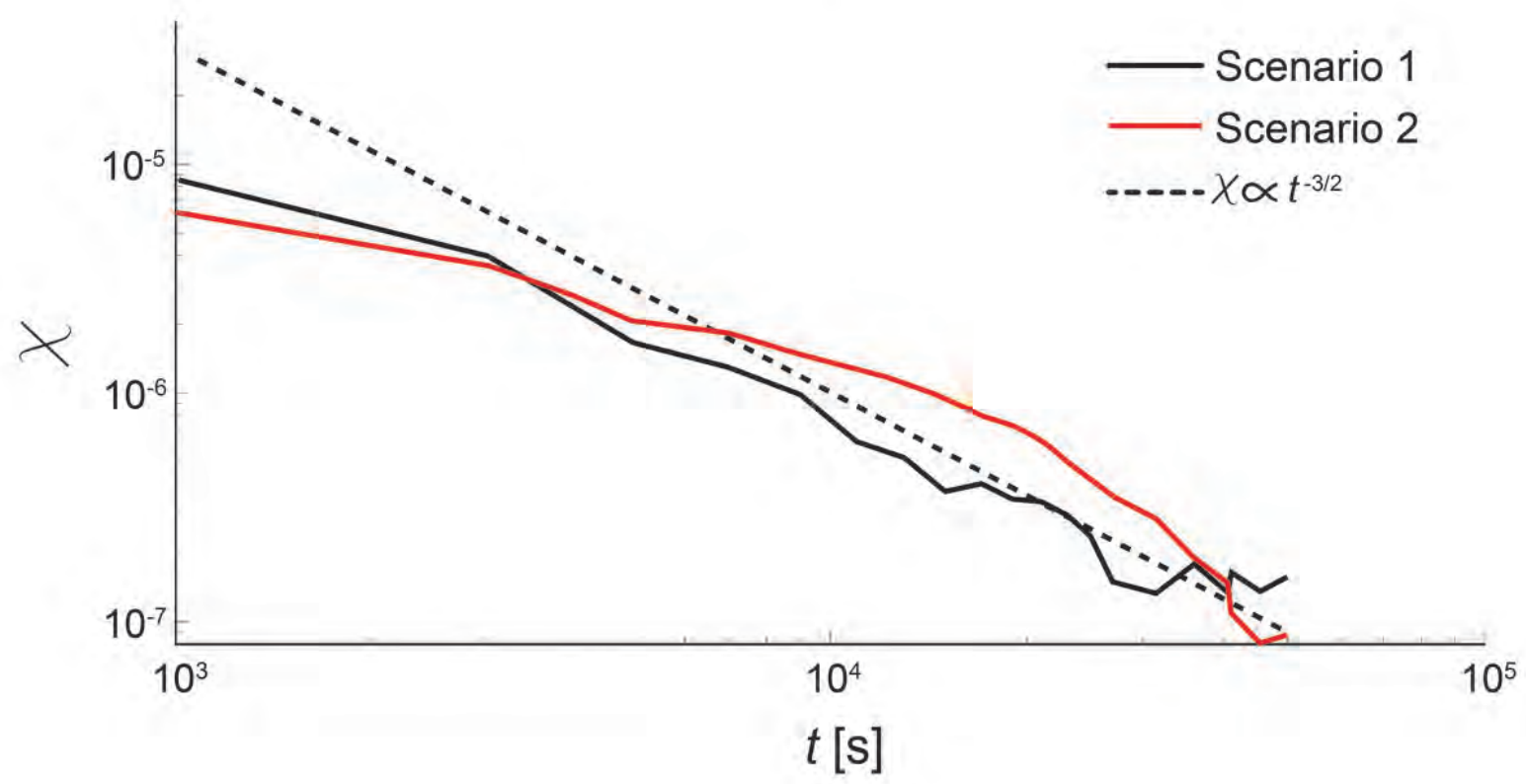

(b) 


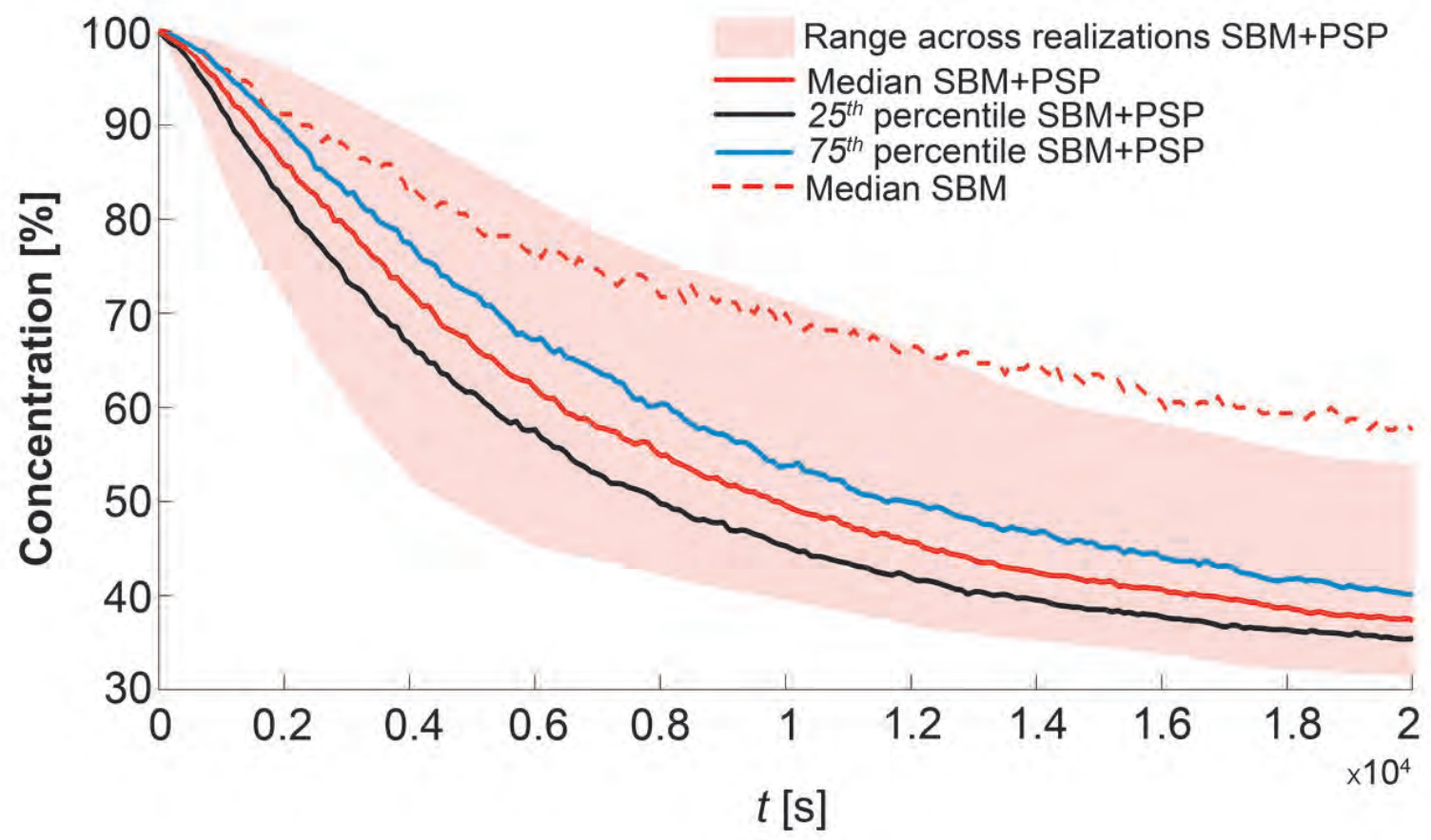

(a)

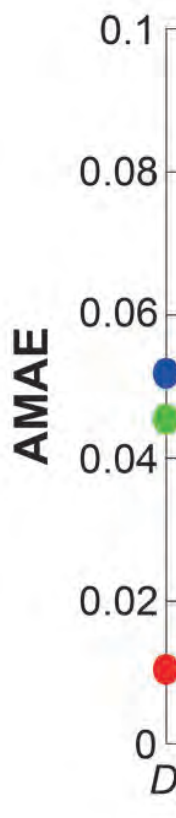

$t=10^{3} \mathrm{~s}$

$t=9.1 \times 10^{3}$

$t=2 \times 10^{4} \mathrm{~s}$

II. 


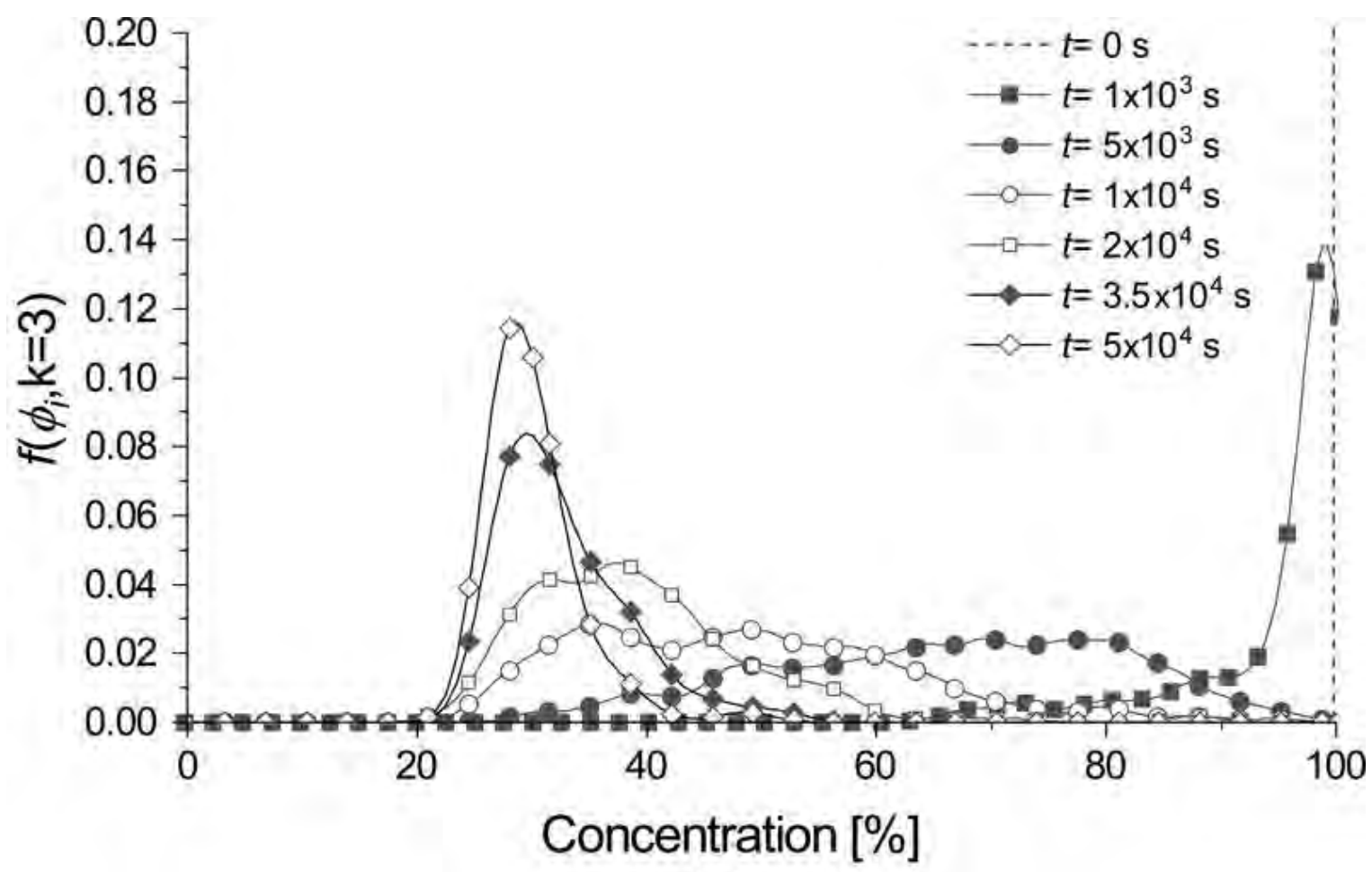

(a)

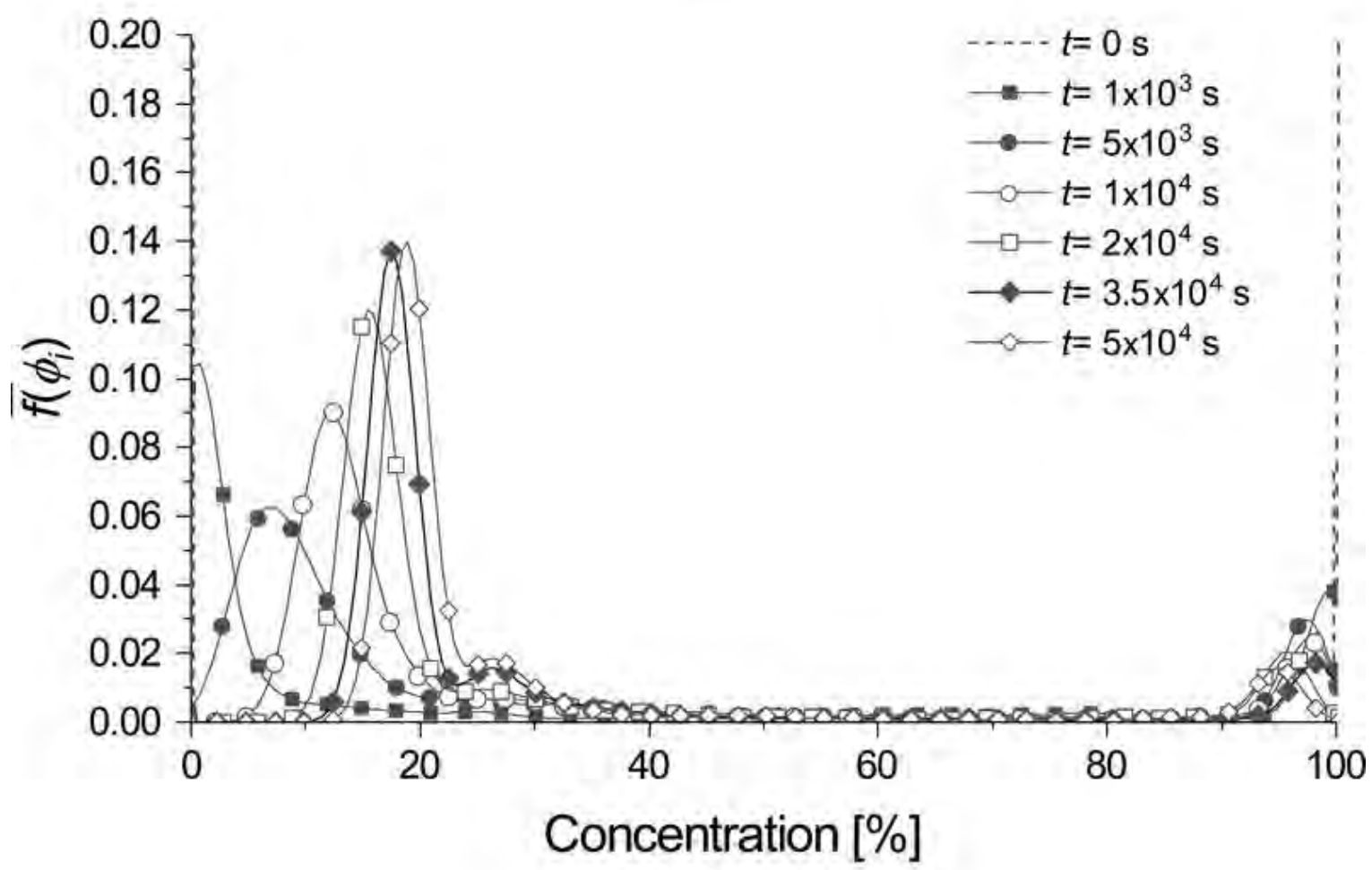

(b) 


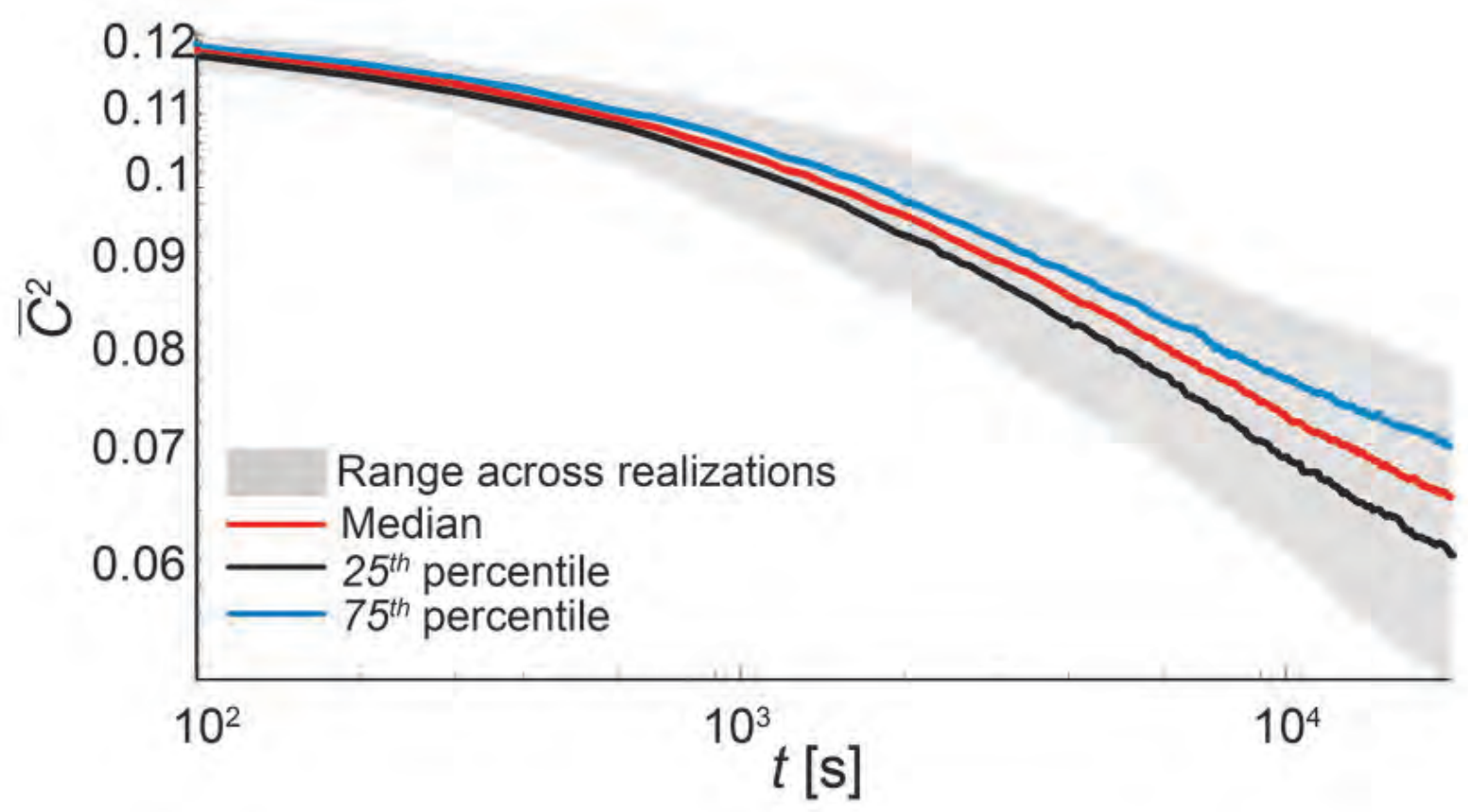

(a)

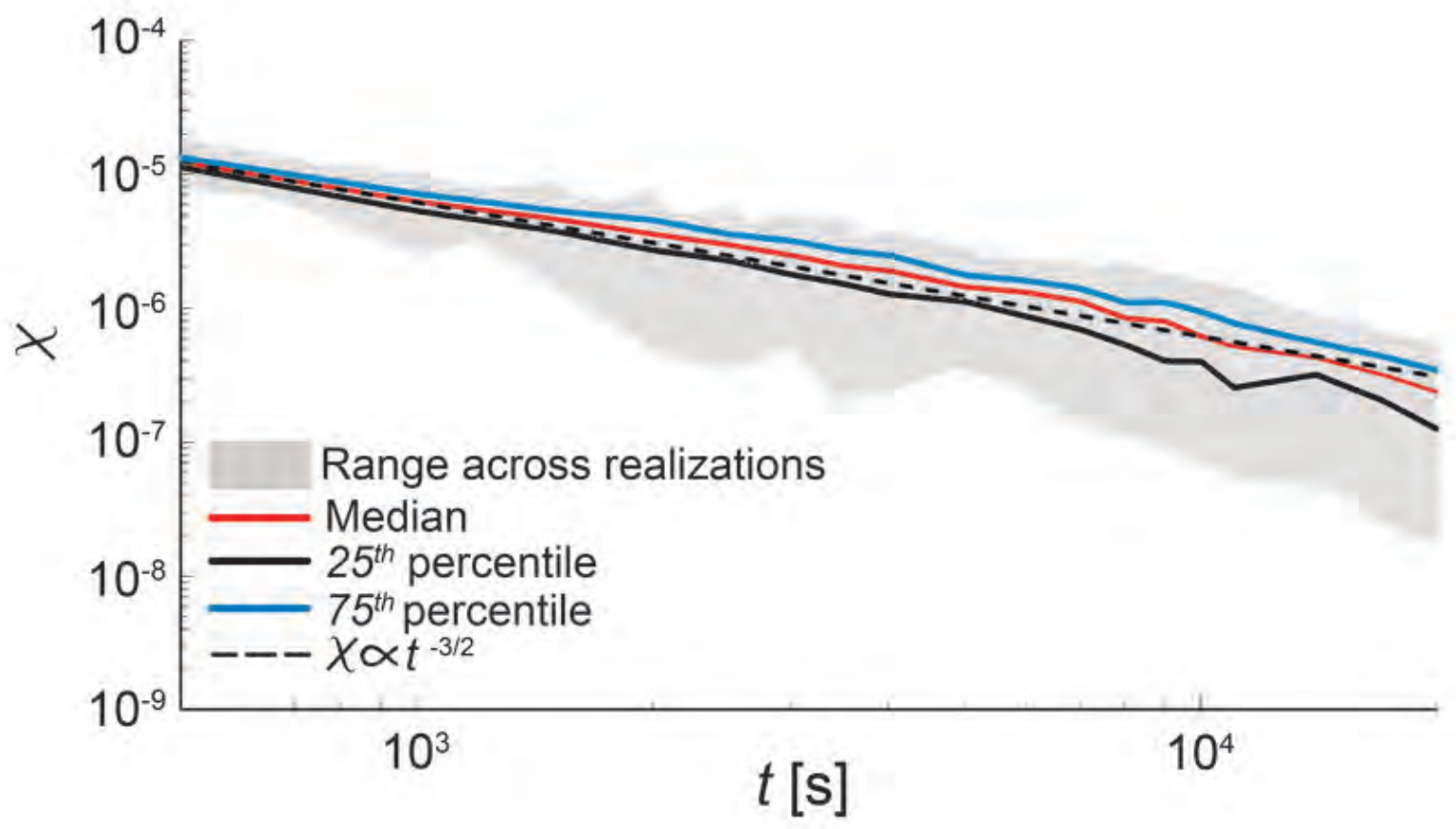

(b) 

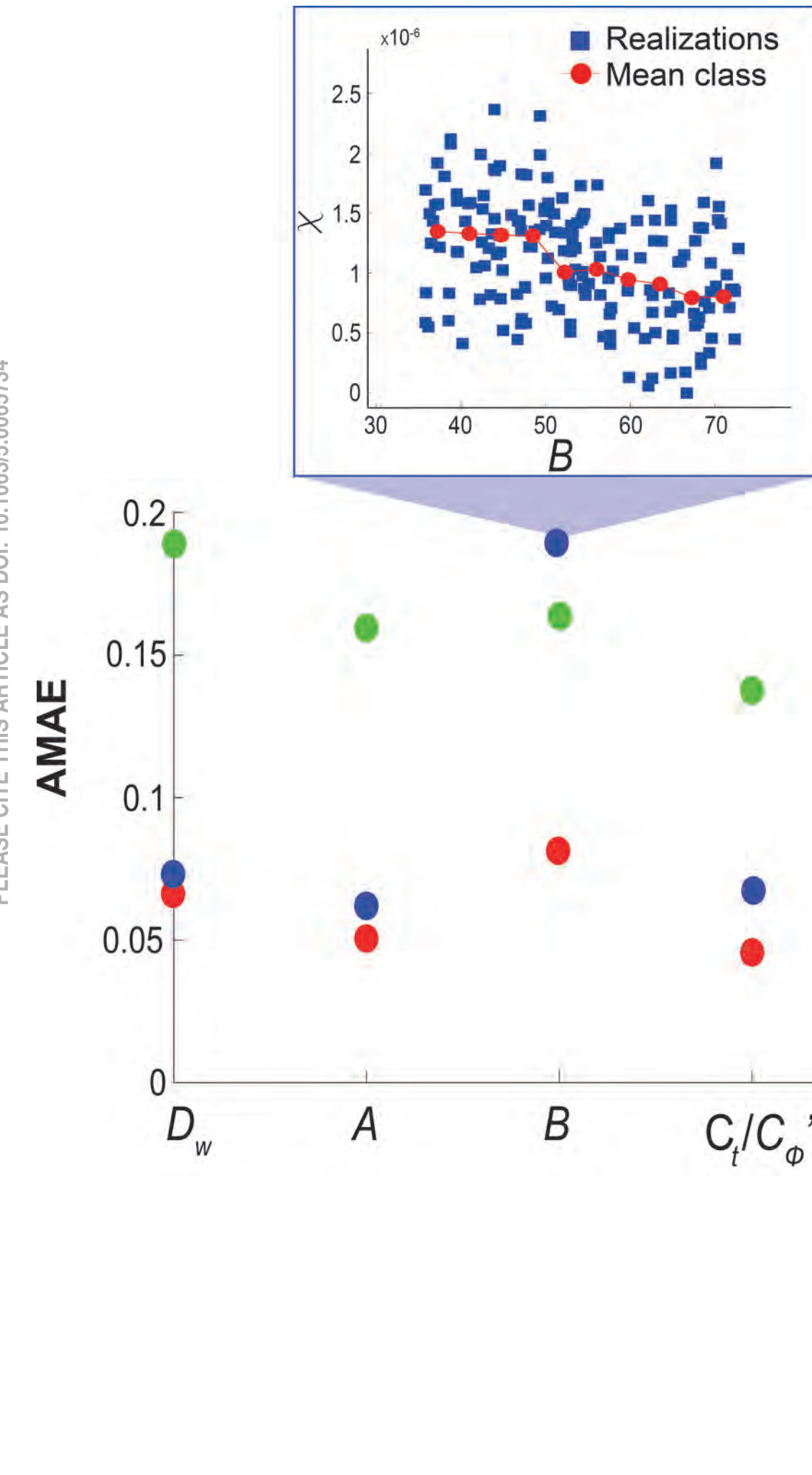